PPPL-2808

PPPL-2808

UC-420, 421, 426, 427

\title{
SIMULATIONS OF DT EXPERIMENTS IN TFTR
}

BY

R. BUDNY, M.G. BELL, H. BIGLARI, ET AL.

December 1991
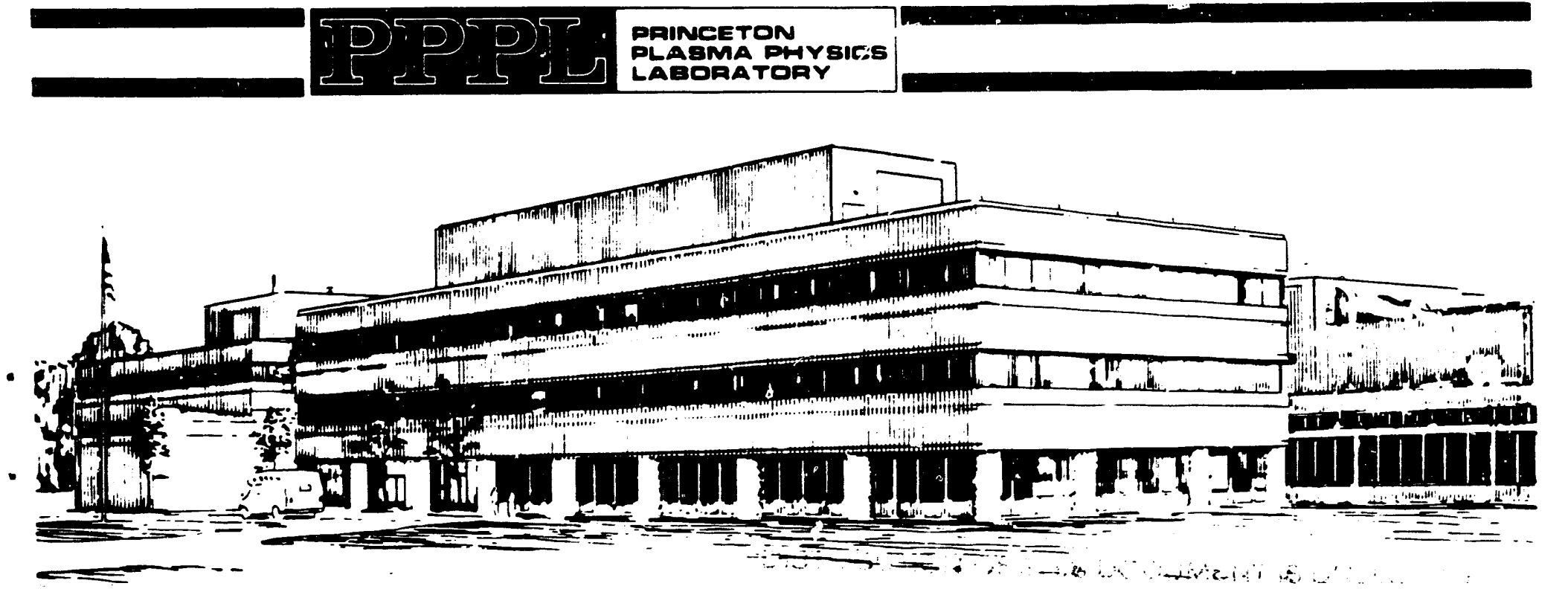

\section{PRINCETON UNIVERSITY, PRINCETON, NEW JERSEY}




\section{NOTICE}

This report was prepared as an account of work sponsored by an agency of the United States Government. Neither the United States Government nor any agency thereof, nor any of their employees, makes any warranty, express or implied, or assumes any legal liability or responsibility for the accuracy, completeness, or usefulness of any information, apparatus, product, or process disclosed, or represents that its use would not infringe privately owned rights. Reference herein to any specific commercial produce, process, or service by trade name, trademark, manufacturer, or otherwise, does not necessarily constitute or imply its endorsement, recommendation, or favoring by the United States Government or any agency thereof. The views and opinions of authors expressed herein do not necessarily state or reflect those of the United States Government or any agency thereof.

\section{NOTICE}

This report has been reproduced directly from the best available copy.

Available to DOE and DOE contractors from the:

Office of Scientific and Technical Information P.O. Box 62

Oak Ridge, TN 37831;

Prices available from (615) 576-8401.

Available to the public from the:

National Technical Information Service

U.S. Department of Commerce 5285 Port Royal Road Springfield, Virginia 22161 703-487-4650 


\title{
SIMULATIONS OF DT EXPERIMENTS IN TFTR
}

\author{
R. Budny, M. G. Bell, H. Biglari, M. Bitter, C. Bush, C. Z. Cheng, E. Fredrickson, \\ B. Grek, K. W. Hill, H. Hsuan, A. Janos, D. L. Jassby, D. Johnson, L. C. Johnson, \\ B. LeBlanc, D. C. McCune, D. R. Mikkelsen, H. Park, A. T. Ramsey, \\ S. A. Sabbagh, S. Scott, J. Schivell, J. D. Strachan, B. C. Stratton, \\ E. Synakowski, G. Taylor, M. C. Zarnstorff, and S. J. Zweben
}

Princeton Plasma Physics Laboratory

Princeton University, Princeton NJ 08543, USA

\begin{abstract}
A transport code (TRANSP) is used to simulate future deuterium-tritium experiments (DT) in TFTR. The simulations are derived from 14 TFTR DD discharges, and the modeling of one supershot is discussed in detail to indicate the degree of accuracy of the TRANSP modeling. Fusion energy yields and $\alpha$-particle parameters are calculated, including profiles of the $\alpha$ slowing down time, average energy, and of the Alfvén speed and frequency. Two types of simulations are discussed. The main emphasis is on the DT equivalent, where an equal mix of $D$ and $T$ is substituted for the $D$ in the initial target plasma, and for the $D^{0}$ in the neutral-beam injection, but the other measured beam and plasma parameters are unchanged. This simulation does not assume that $\alpha$ heating will enhance the plasma parameters, or that confinement will increase with $T$. The maximum relative fusion yield calculated for these simulations is $Q_{D T} \approx 0.3$, and the maximum $\alpha$ contribution to the central toroidal $\beta$ is $\beta_{\alpha}(0) \approx 0.5 \%$.

The stability $c$ f toroidicity-induced Alfvén eigenmodes (TAE) and kinetic ballooning modes (KBM) is discussed. The TAE mode is predicted to become unstable for some of the equivalent simulations, particularly after the termination of neutral beam injection. In the second type of simulation, empirical supershot scaling relations are used to project the performance at the maximum expected beam power. The MHD stability of the simulations is discussed.
\end{abstract}




\section{Introduction}

Experiments using a mix of deuterium and tritium (DT) are planned for TFTR to study plasma conditions near breakeven $\left(Q_{D T} \equiv D T\right.$ fusion power $/ N B \mid$ power $=1$ ), and to study the effects of fusion $\alpha$ particles. The purpose of this paper is to give examples of the fusion energy yields and $\alpha$ parameters which can be expected from the DT experiments in TFTR. Some simulations of DT plasmas in TFTR have already been published. ${ }^{1-5}$ This paper discusses simulations calculated with the TRANSP transport code. $6-8$ Detailed results and profiles are given for use in studying $\alpha$-particle effects and plasma stability.

The TRANSP Monte-Carlo fast ion mode 6 was recently extended to include the fast fusion products from the reactions

$$
\begin{aligned}
& D+D \rightarrow T+p \\
& D+D \rightarrow 3 \mathrm{He}+n \\
& D+T \rightarrow 4 \mathrm{He}+n
\end{aligned}
$$

Monte Carlo births of the $\mathrm{T},{ }^{3} \mathrm{He}$, and ${ }^{4} \mathrm{He}(\alpha)$ are performed using spatially $2 \mathrm{D}$ fusion reaction rate data (beam-beam + beam-target + thermonuclear) computed in the preceding timestep. The fusion products are launched in the (rotating) plasma frame with the appropriate energy (e.g., 3.5 MeV for the $\alpha$ particles). Their orbits, slowingdown, and their heating of the thermal plasma are calculated using the same methods employed in modeling the fast ions from neutral beam injection (NBI). The model takes into account non-zero orbit width effects and Larmor radius effects, but magnetic field ripple effects are not included.

The fusion products slow down and pitch-angle scatter on thermal plasma species (electrons, ions, and impurities). They are treated as thermalized when they slow down to the average energy of the local thermal ion population $\left(\frac{3}{2} k T_{i}\right)$. Collisional coupling between fast ion species (e.g., beam-beam and beam - $\alpha$ collisional effects) is not computed. Effects which are included in the Monte Carlo model of injected fast ions, but-not yet in the fusion products simulation are atomic physics effects (e.g., charge exchange of partially slowed $\alpha$ particles and/or ionization of neutral atoms by $\alpha$ particles), and accumulation of thermalized fusion products. 
The discharges which should show the largest effects from the $\alpha$ particles are those wit'l a high DT fusion rate (the $\alpha$ production rate), with a long slowing-down time for the $r_{\alpha}$ particles, and with a high plasma current to confine the $\alpha$ particles as they theimalize. 4 The fusion rate increases with the products $n_{\text {thermal }}{ }^{2}, n_{\text {beam }} n_{\text {thermal }}$, and $n_{\text {beam }}{ }^{2}$. The $\alpha$ slowing-down time increases approximately as $T_{e}^{1.5} / n_{e}$.

A number of TFTR discharges, listed in Table I, were used for deriving the DT simulations. Most of these are supershots, ${ }^{9}$ chosen since these have the highest neutron rates observed in TFTR. The list includes the discharges with the highest DD fusion rates $\left(55806,55804\right.$, and 53848 with $5.0,4.6$, and $4.4 \times 10^{16} / \mathrm{sec}$, respectively). Three of them had the highest values for $Q_{D D}$ ( $\equiv D D$ fusion power / NBI power). DT simulations were derived from an L-mode discharge with sawteeth, and from a discharge with deuterium pellets injected before the NBI to contrast their yields with tı ose from DT supershots. Deuterium was the dominant ion species for all these discharges.

TRANSP is generally run in a mode which is partly phenomenological and partly predictive. The phenomenological or empirical pari consists of using, as input, measured plasma parameters, such as the total current, the boundary at the last closed flux surface, and temperature and density profiles. There is considerable flexibility in the options for inputing the profiles. For instance, the electron temperature can be input as a profile of electron cyciotron emission (ECE) versus frequency and time, or as concatenations of Thomson scattering (TVTS) profiles at different times versus the major radius.

Parameters can be predicted by TRANSP. For instance, the ion temperature can be predicted by assuming that the ion thermal conductivity, $\chi_{i}$ is a multiple of the electron thermal conductivity, $\chi_{e}$. This modeling assumption is useful when $T_{i}$ has not been measured since it has been found that $\chi_{i}=\left(\begin{array}{lll}1 & \text { or } 2\end{array}\right) \chi_{e}$ predicts $T_{i}$ profiles which are in approximate agreement with measurements in fully-diagnosed supershots. Also the impurity ion temperature, Timp, is predicted by TRANSP from the hydrogenic ion temperature, $\mathrm{T}_{\mathrm{i}}$. The method is described in Appendix $A$. Various other measurements can be predicted or simulated in TRANSP. For instance, chordal integrals of electron densities and visible bremsstrahlung emission can be simulated. 
A sawtooth model, based on Kadomtsev mixing, ${ }^{10}$ is available in TRANSP. Sawtooth crash times are specified as inputs, and TRANSP computes the mixing of the current and fast particles (beam ions and fusion products). The sawtooth model was used for some of the simulations, and has consequences for the $\alpha$ parameters.

This paper concentrates on TRANSP results derived from the supershot 55851. This discharge was selected since it maintained a high DD fusion rate for a long duration, and had a plasma current high enough (1.6 MA) for good $\alpha$ confinement, a high central $T_{e}$ for low electron drag, and extensive diagnostic measurements. Also this discharge was the MHD-free plasma with the largest $Q_{D D}$ measured in TFTR.

Sections 2 - 4 give results from the modeling of 55851. Detailed comparisons of the TRANSP results with measurements are discussed in Sec. 2 in order to indicate the degree of accuracy of the modeling. Alternative modeling assumptions are discussed to indicate the sensitivity to the assumptions. In particular, the neutron emission profile is important since, in the DT simulations, it gives the source rate of the fusion $\alpha$ particles. Calculations of profiles of the pressure and the MHD-q, $q_{\psi}$, needed for the MHD stability analysis, are given. The ideal MHD stability is discussed in Sec. 3. The discharge is calculated to be stable to Mercier modes, ${ }^{11}$ and marginally stable to low- $n$ and high-n modes. A summary of TRANSP predictions for fusion products parameters from the DD reactions is given in Sec. 4. As expected, the parameters are very small compared with the simulated DT parameters.

Two types of DT simulations are then discussed. The main emphasis is on a conservative simulation, called the DT equivalent of actual DD discharges. The second type of simulation is a more optimistic extrapolation of the best present performance to the highest expected NBI power. The DT equivalent simulations are discussed in Sec. 5, and are contrasted with previously published simulations for JET. 12,13 In Sec. 6 , the fusion yields and $\alpha$ parameters for the DT equivalent of 55851 are given. Section 8 presents the results for the DT extrapolation.

For both types of simulations, the measured plasma and NBI parameters from $D D$ discharges are used. The relative $D$ and $T$ densities in the initial plasma, and in the $D^{0}$ and $T^{0}$ beams are assumed to be equal. For the DT equivalent, the measured $n_{e}, T_{e}, T_{i}$, and toroidal rotation profiles are used, along with the measured $Z_{e f f}, N B I$ 
powers, voltages, and full and half energy fractions. For the DT extrapolation, the NBI parameters and the plasma current, temperatures, and densities are scaled up.

The collective $\alpha$-induced TAE and KBM instabilities are discussed in Sec. 8. They are predicted to be marginally unstable for the DT equivalent simulation. For instance, DT equivalent simulations are predicted to pass through the TAE unstable region after termination of NBI. Section 9 gives a summary of the paper.

\section{TRANSP modeling of supershot 55851}

The major and minor radii of this supershot were $R=2.45 \mathrm{~m}$ and $\mathrm{a}=0.8 \mathrm{~m}$, with a toroidal field of $B T F=5.1 \mathrm{~T}$, and $q_{\psi}(\mathrm{a})=5.5$. A Li pellet was injected into the target plasma early (at $2.0 \mathrm{sec}$ ) to improve the plasma performance. ${ }^{14} \mathrm{Li}$ pellets reduce the carbon concentration in supershots, apparently by coating the limiter, reducing carbon sputtering. The NBI power was $25 \mathrm{MW}$. The total current, lp was increased in the early phase of the NBI, as shown in Fig.1A. This increase was programmed in an attempt to improve the MHD stability. This discharge, like most supershots with plasma currents below about 1.8 MA, did not have sawteeth during the NBI phase. The normalized toroidal- $\beta$, defined by

$$
\beta_{\text {norm }} \equiv \frac{\left\langle\beta_{\text {tor }}>\right.}{I_{\mathrm{p}} /\left(\mathrm{aB} \mathrm{B}_{\mathrm{TF}}\right)},
$$

reached 2.25. < > designates the volume average, and $\left.\beta_{\text {tor, }}\right|_{p}, a$, and $B_{T F}$ are in \%, $M A, m$, and $T$. The peak value of $\beta_{\text {norm }}$ is below the highest value achieved in supershots (2.7). The total energy confinement time reached a peak of $0.17 \mathrm{sec}$ around the time of maximum neutron emission.

For the TRANSP modeling of 55851, various combinations of inputs for the temperatures were investigated. For instance, the electron temperature was derived in TRANSP either from an absolutely-calibrated, time-dependent profile of first harmonic ECE measured by a radiometer, 15 or derived from the time-dependent profile of second harmonic ECE measured by a grating polychromator ${ }^{16}$ (GPC). In the latter case, the profile was cross-calibrated either by the second harmonic ECE measured by a Michelson interfermeter, 17 or by the TVTS profile ${ }^{18}$ at $3.46 \mathrm{sec}$. The central 
electron temperatures from the first two methods are about $15 \%$ higher than the values from GPC cross-calibrated by TVTS, peaking above $11 \mathrm{KeV}$. Differences of this magnitude are typically measured in supershots with high $T_{e}$ (above about $8 \mathrm{KeV}$ ).

All three derivations of $T_{e}$ were modeled in different TRANSP runs. Comparisons of the modeling results with other measured parameters could not definitively exclude these alternative derivations. We indicate the sensitivity of our results to the assumptions about $T_{e}$, and focus on the derivation using GPC, crosscalibrated by TVTS.

In the usual TRANSP modeling of supershots, the hydrogenic ion temperature, $T_{i}$, is fit to measured temperature profiles of impurity ions. The impurity temperature, Timp, is higher than $T_{i}$, and TRANSP was modified, as discussed in Appendix $A$, to estimate this difference. We modeled these corrections by fitting Timp to the timedependent temperatures derived from charge-exchange recombination spectroscopy ${ }^{19}$ (CHERS) of emission from the $n=8 \rightarrow n=7$ transition in the hydrogenlike carbon impurity. These measurements were available only for the NBI phase of the discharge, so the assumption $\chi_{i}=2 \chi_{e}$ was used for the ohmic phases. The central values of the TRANSP results for $T_{i m p}$ and $T_{i}$ are shown in Fig. 1B. The differences are relatively large at the start on NBI, and decrease with time.

Time-dependent profiles of Timp were also calculated from measurements with an X-ray crystal spectrometer 20 of Doppler broadening of the resonance $K \alpha$ emission from the helium-like iron impurity. Comparisons of the computed and measured temperature profiles at the TVTS time are shown in Fig. 1C. The differences between the two measurements of Timp shown here are atypically large, and are less at later times in the discharge.

The toroidal rotation velocity of the carbon was measured by CHERS and input into TRANSP as the rotation velocity of the thermal plasma. This velocity is relatively large (peaking at $5 \times 10^{5} \mathrm{~m} / \mathrm{sec}$ ), even though the NBI was approximately balanced with equal co and counter tangential injection (relative to the direction of the plasma current). 
The electron density was smoothed and symmetrized from the time-dependent Abel-inverted profiles measured by a 10 chord far infrared interferometer system. 21 (MIRI). A comparison of the measured and calculated profiles at the TVTS time are shown in Fig. $1 \mathrm{D}$.

The surface voltage was calculated using neoclassical resistivity and the beamdriven and bootstrap currents. 22 The result compares well with the measured value, as shown in Fig. $1 E$. For the modeling using the higher $T_{e}$ derived from the ECE measured by GPC, the agreement between the measured and calculated voltage in was not as good in the ohmic phase. Zeff was calculated from the visible bremsstrahlung signal, and the result is shown in Fig. $1 F$. The impurity radiation was measured with a multichannel grazing-incidence spectrometer. 23 The dominant impurity in this discharge was carbon. $Z_{\text {met }}$, the metallic contribution to $Z_{\text {eff }}$, was measured with $X$-ray pulse height analysis 24 (PHA), and is shown as well.

Much of the increase in Zeff during NBI is due to the increasing concentration of metallic impurities. Since TRANSP allows only one impurity species, the impurity was chosen to have $Z=7.8$ instead of 6 . This gives the correct depletion of the deuterium density corresponding to the measured $Z_{\text {eff }}$ and $Z_{\text {met }}$ around the time of maximum neutron emission. The calculated neutron emission rate depends sensitively on the $D$ depletion. The hydrogenic recycling rate, which was input into TRANSP, was computed from five chordal signals of $D_{\alpha}$ emission using the 3D neutrals code DEGAS.?5

The perpendicular energy calculated by TRANSP is compared with the diamagnetically measured value in Fig. $1 G$. They agree within the estimated errors. The measured value was shifted up by $80 \mathrm{KJ}$ to match the calculated values in the preNBI phase, where the measured value results from the subtraction of two measured quantities of comparable magnitude. TRANSP modialing using the higher $T_{e}$ from ECE yielded perpendicular energies which were also in yood agreement with the measured values. TRANSP modeling using the usual assumptions where $T_{i}$ instead of Timp is fit to the ion temperature measurements yielded a peak perpendicular energy that is $100 \mathrm{KJ}$ higher than the result given here with the Timp correction.

The calculated DD neutron emission rates are shown in Fig. $1 \mathrm{H}$. The total rate is within the absolute error bars of the measurements from a calibrated neutron 
diagnostic. 26 The beam-target contribution is $55 \%$ of the total rate. An independent calculation of the neutron rates was done with the 1D, time-independent code SNAP, 27 and the results are shown in Fig. $1 \mathrm{H}$ also. The TRANSP modeling using the usual assumptions with $T_{i}$ fit to the impurity ion temperature measurements yielded a peak neutron rate that is approximately $5 \%$ higher. TRANSP modeling using the higher $\mathrm{T}_{e}$ from ECE yielded neutron emission rates which were again approximately $5 \%$ higher. The sensitivity of the modeling to the assumed fusion cross section fits was investigated. Until recently, the fits of Duane 28 had been used in TRANSP. When the more recent fits of Bosch 29 were used, the total neutron rate decreased about $5 \%$.

An array of independently-calibrated collimated neutron detectors 30 was also used to measure neutron emission rates. The chord-integrated signals were simulated with TRANSP. The simulated rates are consistent with the measured rates, as shown in Fig. 1L. The collimators are separated by approximately $0.2 \mathrm{~m}$ in major radius, so this comparison is not a precise check of the location of the peak of the neutron emitting region, or of the width of the profile. The raw data from the collimated detectors includes contributions from neutrons scattered from the tokamak and the surrounding structure, which have been estimated and subtracted from the measurement. The reiative error from this contribution is higher near the edge of the plasma, where it is comparable to the net result. A more precise indication of the plasma center is given by the location of the peak of the soft $X$-ray emission. 31,32 This is compared with the TRANSP calculation in Fig. $1 \mathrm{~J}$, and agrees to within several centimeters.

The Kadomtsev sawtooth model was used in the modeling of the pre- and postNBI sawteeth. This alters the plasma current, and raises $q_{\psi}(0)$ to 1 at the sawteath events. Profiles of $q_{\psi}$ and of the shear, defined as

$$
s \equiv \frac{d \ln q \psi}{d \ln x}
$$

are shown in Fig. 1K. The profiles are plotted versus the normalized toroidal flux coordinate, $x \equiv$ the square root of the toroidal flux normalized to the value at the plasma boundary. The vaiue of this variable is very ciose to the normalized minor radius ( $r / a$ ). Profiles of the thermal and total pressure (beam plus thermal) are shown in Fig. 1L. 


\section{MHD activity and stability of supershot 55851}

There was no observed coherent MHD activity in this discharge. In contrast, very similar discharges developed severe MHD problems as early as $0.3 \mathrm{sec}$ following the start of NBI. The MHD modes in those cases were $(m, n)=(2,1)$ and $(3,2)$ modes. 33 The onset of these modes coincided with a severe degradation of the confinement and the neutron production rate. These modes are thought to be resistive in nature due to their long growth rates. It is believed that the stability of these modes could depend very sensitively on very small differences in the pressure and $q_{\psi}$ profiles. We do not understand why som: discharges do, and others do not exhibit coherent MHD, and a clear prescription for avoiding MHD in supershots has not been found.

The calculated $q_{\psi}$ and total pressure profiles (including beam pressure) were used to study the ideal MHD stability of this supershot. The high-n stability was investigated using the EQGRUM 34 and STBAL 35 equilibrium codes. Figure 2 shows the profile of the gradient of the total pressure with respect to the normalized poloidal flux, along with the boundary of the unstable region. Both are plotted versus the square-root of the normalized poloidal flux, $\psi$. The profiles are calculated to be stable to Mercier modes, and marginally stable to high- $n$ ballooning mc Jes near $x=0.5$.

The low-n stability was analyzed using the PEST 2 code. 36 The plasma was found to be marginally stable to an $n=1$ free-boundary (conducting wall at infinity) kink/ballooning mode. The marginal stability value of $\beta$ norm is computed to be in the range $2.30-2.38$, which is just above the actual value for the discharge (2.25). If the pressure is raised such that $\beta$ norm exceeds this value, the $n=1$ external kink/ballooning mode is predicted to be unstable.

\section{TRANSP simulation of fusion products from $\mathbf{5 5 8 5 1}$}

The TRANSP fusion products calculations were performed for 55851, using the Monte Carlo orbit model described in Sec. 1. The peak central densities of the unthermalized fusion products ( $f p$ ), as a fraction of the electron density during NBI, are listed in Table II. The fraction increases to higher values after termination of NBI since the thermal particle confinement time is short compared with the fp slowing-down time, and thus $n_{e}(0)$ decreases faster than $n_{f p}(0)$. 
Another parameter of interest is the contribution of the fp pressure or $\beta_{f p}$ to the total pressure or toroidal- $\beta$. Results for $T$ and $3 \mathrm{He}$ from the $D D$ fusion reactions are summarized in Table II. Still another parameter of interest is the ratio of the birth speed of the fusion products and the central Alfvén speed. The birth speed is $7 \times 10^{6}$ $\mathrm{m} / \mathrm{sec}$ for the ${ }^{3} \mathrm{He}$ and $8 \times 10^{6} \mathrm{~m} / \mathrm{sec}$ for the T. The Alfven speed is given by

$$
v_{\text {Alfvén }}=\frac{B_{T F}}{\sqrt{4 \pi m_{H}\left(2 n_{D}+n_{H}+2 n_{\text {beam }}+15 n_{\text {imp }}\right)}}
$$

where the average atomic mass of the impurities is approximated by 15 , and the very small contribution from the fusion products is neglected. The peak central values of the ratio are given in Table II. They are of interest for the TAE instability, which is discussed in Sec. 8.

The slowing down time and average energy of the ${ }^{3} \mathrm{He}$ ions are also calculated. The average energy of the $3 \mathrm{He}$ ions decreases from the birth energy of $0.8 \mathrm{MeV}$ to 0.4 $\mathrm{MeV}$ late in the NBI phase. The slowing down time also decreases from 0.5 to $0.2 \mathrm{sec}$ during NBI.

\section{General results from the equivalent simulations}

The DT equivalent for each DD discharge in Table | was calculated using minimal changes in the TRANSP inputs for the DD modeling. The assumptions used for the modeling of the DD discharges differed, however, depending on available measurements. One major difference was whether $T_{i}$ profile measurements were available. For the discharges that did not have measured profiles, the assumption $\chi_{i} \approx$ $2 \chi_{\theta}$ was used to calculate $T_{j}$. This assumption adds uncertainty to the results for these discharges, and makes comparisuns between discharges more difficult.

The plasma densities are calculated in TRANSP using the particle balance equations. The electron density is symmetrized from the measured profile, so conservation of electrons determines the local radial flux of electrons. The values of $Z_{\text {eff }}$ and the conservation of the impurity ion species determine the radial flux of the impurity. The $D$ and $T$ sources are determined by the NBI deposition profiles and by the $D$ and $T$ recycling. To solve the conservation equations for $D$ and $T$, the relative 
fractions of the total $N_{D}$ and $N_{T}$ in the initial (pre-NBI) plasma at the start of the calculation must be specified, and the relative radial fluxes of the $D$ and $T$ must be specified. Two specifications for the relative radial fluxes were investigated. For the cases listed in Table I, the radial velocities of the $D$ and $T$ were assumed to be equal. An alternative choice, where the $D$ and $T$ diffusivities are assumed to be equal, gives similar results.

For simplicity, he only differences in the inputs for the DT equivalent sirnulations were 1) the change in the hydrogenic fractions of the initial (pre-NBI) plasma from typically $95 \% \mathrm{D}$ and $5 \% \mathrm{H}$ to $50 \% \mathrm{D}$ and $50 \% \mathrm{r}, 2$ ) the change in recycling from $95 \% D^{0}$ and $5 \% H^{0}$ to $50 \% D^{0}$ and $50 \% T^{\circ}$, and 3 ) the duplication of the NBI $D^{0}$ sources as $T^{0}$ sources, with the original powers split equaily between the $D^{0}$ and $T^{0}$ sources. This procedure preserves the co - counter split of the NBI.

The total hydrogenic recycling rates were not changed from those of the $C D$ discharges, so assumption 2) means that the rates of $D$ and $T$ recycling are each haif the rate measured for the $D D$ discharges. This models the case where the limiter would be saturated equally with both $D$ and $T$. For the $D T$ run scenarios planned for TFTR, the limiter is not expected to be saturated with $T$, so the hydrogenic recycling will be mainly $D$. Also $T$ gas puffing is not planned, so the pre-NBI target plasmas for supershots is expected to be mainly $C$ with some $D$.

DT simulations with the alternative assumptions of relatively little $T$ in the target plasma and in the recycling have been performed. The results for supershots are that the neutron yield and $\alpha$ parameters are insensitive to the assumptions 1) and 3 ). This is because most of the fueling of the central region is from the NBI. The beam deposition profiles of the $D$ and T NBI are similar, and the resulting central densities are predicted to be approximately equal. The total neutron emission rate depends mainly on the central $D$ and $T$ densities, but does not depend sensitively on the precise matching of the central $D$ and $T$ densities. In $D T$ supershots, the $T$ density in the plasma edge is expected to be lower than that of $D$ due to the reduced $T$ recycling.

The values of $Q$ for each $D T$ / DD pair were calculated by dividing the peak fusion power yields by the injected NBI power. The total fusion power (thermonuclear + beam-taiget + beam-beam) was used. The calculated neutron rates were converted to fusion power using $17.6 \mathrm{MeV}$ / neutron for DT, and 7.3 MeV/ neutron for DD. No 
reductions of the input power, such as those due to beam ion orbit loss or beam shinethrough are included. The resulting $Q$ values and their ratios are listed in Table 1. Most of the calculated values for $Q_{D D}$ agree with the experimental values to within the uncertainties of the neutron emission measurements. Both the measurements and the calculations normalize with the same NBI powers, so the uncertainty in the power does not enter in the uncertainty in $Q_{D D}$.

The ratios of the predicted $Q_{D T} / Q_{D D}$, given in Table 1 , are of interest for indicating the scaling from DD to DT yields. Some of the systematic errors in the predictions cancel in this ratio, so that it is more reliably predicted than either $Q_{D D}$ or $Q_{D T}$ separately. The predicted ratios for most of the supershots in Table I lie between 150 and 170. Most of the variation in the ratio comes frorn variation in the $T_{i}$ for the discharges. The $Q_{D T} / Q_{D D}$ ratio decreases with $T_{i}$ since $Q_{D D}$ increases more rapidly with $T_{i}$ than does $Q_{D T}$.

Independent calculations of $Q_{D D}$ and $Q_{D T}$ for the discharges in Table I were made with the SURVEY code. This is a steady-state, fixed profile simulation code which uses the plasma parameters in the TRANSP analysis. It does not include any drift orbit effects (i.e., orbit spreading and orbit losses) in the modeling of fast ions or fusion products. The SURVEY results agree with the values from TRANSP to $\pm 5 \%$. SURVEY was also used to determine the effects of two minor improvements not, th incorporated in most of the TRANSP simulations in Table 1: 1) using the more accurate fusion cross section fits of Bosch 28 instead of those of Duane, 29 and 2) using the full and half-energy fractions expected for $T$ NBI instead of the $D$ fractions. The net effect of the corrections on the ratio $Q_{D T} / Q_{D D}$ is an increase of $(5 \pm 5) \%$ above the TRANSP values. The SURVEY result for the experimentally projected $Q_{D T}$, defined as the product of the calculated $Q_{D T} / Q_{D D}$ times the measured $Q_{D D}$ is plotted versus the measured $Q_{D D}$ in Fig. 3.

Predictions of neutron yields for DT experiments in JET have been published.12,13 The simulations in these papers are not DT equivalents of DD discharges since the beam voltages were increased (to $140 \mathrm{KeV}$ for the $D$ and 160 $\mathrm{KeV}$ for the $\mathrm{T} \mathrm{NBI}$ ). Also the definition of $\mathrm{Q}$ in these papers is not the same as the one used here since the absorbed beam power minus the rate of increase of the total energy ( $d W / d t)$ is used instead of the full NBI power for the thermonuclear contribution to the total neutron yield. The predictions for QDT using the JET definitions are in the 
range 0.7 ? -0.76 . If these corrections for NBI power are not included, as is the case of the TFTR values predicted here, the $Q_{D T}$ values are estimated to be $0.51-0.54$.

Results for the peak values of $\beta_{\alpha}(0)$ and $\left\langle\beta_{\alpha}>\right.$ are also shown in Table I. Peak values for the ratio $n_{\alpha}(0) / n_{e}(0)$ during the NBI phase of the discharges are close to the values of $\left\langle\beta_{\alpha}>\right.$ (typically within $15 \%$ ).

\section{Fusion yields and $\alpha$ parameters from the DT equivalent simulation of 55851}

Results from the DT equivalent simulation of 55851 are summarized in Fig. 4. The neutron rates are shown in Fig. 4A. The peak beam-target contribution is $55 \%$ of the total, as in the DD result (Fig. $1 \mathrm{H}$ ), and the peak thermonuclear contribution is $30 \%$ of the total. The total DT neutron rate is the $\alpha$ source rate. Profiles of the components of the DT fusion emission are shown in Fig. 4B. Besides the DT reaction [Eq. (1c)], TRANSP computes the DD fusions [Eqs. (1a-b)] and TT fusions with much lower rates. The total rate gives $Q_{D T}=0.28$, which is close to the highest values shown in Table I.

Profiles of densities at the end of the NBI are shown in Fig. 4C. The fast $\alpha$ density is much more peaked than the electron density. The central value is $0.3 \%$ that of the electron density and more than an order of magnitude below the impurity density. After NBI, the decrease in the $\alpha$ density is not as rapid as the decrease in the electron density, since the $\alpha$ slowing down time is long relative to the thermal particle confinement time.

The volume integrated heating powers are shown in Fig. 4D. Most of the $\alpha$ heating is to the electrons, $\mathrm{P}_{\alpha->e}$. This is overshadowed by the NBI heating during the NBI phase, and by the ohmic heating after the NBI. The $\alpha$ heating of ions, $P_{\alpha->i o n}$, is lower than $P_{\alpha \text {->e }}$ by nearly an order of magnitude. The predicted orbit loss of $\alpha$ particle power to the limiter and walls is less than $10 \%$ of the total $\alpha$ heating rate.

The proximity to central ignition 3 can be indicated by the ratios of the $\alpha$ heating and power loss terms, 


$$
\frac{\int_{0}^{x} P_{\alpha \rightarrow j} d V}{\int_{0}^{x} P_{j \rightarrow \text { loss }} d V}
$$

(for $\mathrm{j}=$ thermal ions, electrons, and both), where the loss channel for electrons is convection, conduction, and radiation, and for ions is convection, conduction, and net charge exchange. The ion-electron coupling would add to the ion loss and subtract from the electron loss, canceling in the total thermal loss. These ratios are shown in Fig. 4E. Central ignition would occur if the ratio for the thermal plasma were greater than 1 in the vicinity of $x=0$.

The central values of the various contributions to the toroidal $\beta$ are shown in Fig. $4 \mathrm{~F}$. Also the volume averaged value of $\beta_{\alpha}$ are shown. The peak value, $\beta_{\alpha}(0)=0.3 \%$, is within the range projected in Refs. 1,2 , and $4(0.16-0.50 \%)$, and is comparable to the highest values given in Table I. Profiles of the contributions to the toroidal $\beta$ near the time of peak $\beta$ are shown in Fig. $4 \mathrm{G}$. Relative profiles for the $\alpha$-density, $\beta \alpha$, and heating rates near the end of NBI are shown in Fig. $4 \mathrm{H}$. These profile shapes are very similar to each other, except that the $\alpha$ heating of electrons is slightly broader, and the $\alpha$ heating of ions is slightly narrower than the others.

Profiles of the slowing-down time and the average energy of the $\alpha$ particles are shown in Fig. 4l. The value of the central slowing down time, is $\tau_{\text {slow }}(0) \approx 0.4 \mathrm{sec}$ between 3.6 and $3.9 \mathrm{sec}$. The average energy in the central region drops slowly, reaching an approximately steady-state value by the end of NBI. The energy distribution function is thus not in steady state at early times, or even at the time of peak neutron production.

The Alfvén speed for the DT simulations is given, by

$$
v_{\text {Alfvén }}=\frac{B_{T F}}{\sqrt{4 \pi m_{H}\left(2 n_{D}+3 n T+2.5 n_{\text {beam }}+4 n_{\alpha}+15 n_{\text {imp }}\right)}} .
$$


The values given by this expression differ slightly from the values given by Eq. (4) for the DD reaction due to the different masses of the ions. The profile of the flux surface average of this speed is shown in Fig. 4J. Also the profile of the flux surface average of the Alfven frequency, estimated 37,38 by

$$
\omega_{\text {Alfvén }} \approx \frac{\text { VAlfvén }}{2 q \psi^{R}}
$$

is given in Fig. 4J. The ratio of the $\alpha$ birth speed $\left(1.3 \times 10^{7} \mathrm{~m} / \mathrm{sec}\right)$ to that of the fluxaveraged Alfvén speed is shown in Fig. 4K. Implications of these parameters are discussed in Sec. 8.

The results from this particular equivalent simulation are fairly representative of the results from the best supershots listed in Table I.

\section{DT extrapolations}

There are reasons to suspect that the actual DT plasma performance in TFTR might be better than that indicated by the equivalent simulations. A DT extrapolation is described to provide an example of neutron yields and $\alpha$ parameters which potentially will occur, and also to indicate what improvemients would be required to enhance the yield and $\alpha$ parameters. A DT plasma with the same setup and NBI parameters as 55851 could be expected to perform better than the DD plasma, due to potential favorable mass scaling of confinement, and due to $\alpha$ heating of electrons. The equivalent simulations use the actual measured profiles, so they do not claim the advantage of these possibilities.

The NBI power was relatively low for 55851 (25 MW) compared to the highest achieved in TFTR (33 MW). If the MHD and $Z_{\text {eff }}$ can be controlled, then the neutron yields and $\alpha$ parameters should increase as the heating improves. As the power increases, the plasma density and energy increase, giving a higher neutron yield. Despite this, the higher power supershots listed in Table I did not have substantially better $Q_{D T}$ yields. However, with the accumulation of more operating experience, better performance may be expected. 
The extrapolations were clerived by scaling the 55851 parameters to higher power using empirical scaling relations which have been derived from statistical analysis of more than 550 TFTR supershots from 1990.39 These scaling relations give the central plasma paramieters at the time of maximum neutron emission, $n_{e}(0), n_{D}(0)$, $T_{e}(0)$, and $T_{D}(0)$, as single products of powers of $B_{T F}, P_{N B \mid}, l_{p}, E_{N B \mid}$, and $\tau_{E}{ }^{\text {total }}$. $E_{N B I}$ is the energy of the full energy component in the NBI. The total energy confinement time, $\tau_{E}{ }^{\text {total }}$, is given as a product of powers of $B_{T F}, C_{\text {target }}, I_{p},\left(1+F_{N B I}\right)$ and $E_{N B l}$, where $C_{\text {target }}$ is the average $C$ II emission in the target plasma jusi before $\mathrm{NBI}$, and $\mathrm{F}_{\mathrm{NBI}}$ is the fraction of the $\mathrm{NBI}$ power in the co-direction.

Not all the parameters for 55851 were predicted well by the empirical scaling relations due, probably, to the Li pellet injection and the current ramp. The values of $n_{e}(0)$ and $n_{D}(0)$ were $40 \%$ higher than the values given by the empirical scaling relations, whereas the values of $T_{e}(0), T_{D}(0), \tau_{E}{ }^{\text {total }}$ and the peak neutron rate were consistent. A DD extrapolation of 55851 was derived by increasing $P_{N B I}, l_{p}$ and $C_{\text {target}}$, using the scaling relations to give multipliers of $n_{e}(0), n_{D}(0), T_{e}(0), T_{D}(0)$ and $\tau_{E}{ }^{\text {total }}$. This scaled DD supershot was converted to a DT supershot, incorporating some of the anticipated $\alpha$ heating of electrons.

The NBI power was increased from 25 to $35 \mathrm{MW}$, and the plasma current was increased by a factor of 1.2 to a peak value of $1.95 \mathrm{MA}$, which is close to the present empirical upper limit for achieving supershots in TFTR. The scaling variation with $I_{p}$ is not favorable, but $I_{p}$ was increased to keep $\beta$ norm from surpassing the present empirical limit for supershots (around 2.7). BTF was held constant, although the scaling relations predict a rapid improvement of plasma performance with increasing $B_{T F}$. $F_{N B I}$ and $E_{N B I}$ were kept near their values for 55851 (approximately 0.57 and $105 \mathrm{KeV}$ ), even though the scaling relations favor a decrease of $\mathrm{E}_{\mathrm{NBI}}$.

With $P_{N B \mid}, I_{p}, B_{T F}, E_{N B \mid}$, and $F_{N B \mid}$, as chosen above, the scaling of $n_{e}(0)$, $n_{D}(0), T_{e}(0)$, and $T_{D}(0)$ are determined solely by $\tau_{E}{ }^{\text {total }}$, which decreases weakly with increasing $C_{\text {target }}$. We assumed that $\tau_{E}{ }^{\text {total }}$ would remain at the value observed for $55851(0.17 \mathrm{sec})$. The scaling relations requires that $C_{\text {target }}$ would increase by $15 \%$. An increase of $C_{\text {target }}$ with increased NBI power would be expected due to the increased power loading on the limiter. With these choices of parameters, the scaling relations imply that $n_{e}(0)$ and $n_{D}(0)$ would increase by $15 \%$. The latter two quantities 
determine Zeff, which would be slightly below the values observed in 55851 . The scaling of $T_{D}(0)$ results in a $7 \%$ increase, and that of $T_{e}(0)$ results in a $4 \%$ increase.

To convert the DD extrapolation to a DT extrapolation, we assume, as in the equivalent simulations, that $n_{D}$ is replaced with a 50/50 mix of $n_{D}$ and $n_{T}$ in the pre$N B I$ target, and that the NBI is split equally with $D$ and $T . T_{e}(0)$ could be expected to be higher than indicated by the DD scaling relations due to the $\alpha$ heating and possibly due to favorable isotope scaling. In order to incorporate the $\alpha$ heating, we consider the ratio

$$
\frac{\int_{0}^{x} P_{\alpha \rightarrow e} d V}{\int_{0}^{x}\left[P_{\text {beam } \rightarrow e}+Q_{i e}\right] d V}
$$

where $Q_{i e}$ is the ion $\rightarrow$ electron heat coupling. This ratio obtains high values outside $x=0.25$ (the half radius of the neutron emission profile), as shown in Fig. 5A. Even at $x=1$, the ratio is 0.24 . This increased heating could result in an increase in $T_{e}$, or a loss in energy confinement (increase in $\chi_{e}$ ), or both.

To compromise, we assume that $T_{e}$ will increase by $21 \%$ more than the amount given by the DD scaling of 55851 , i.e., by a total of $25 \%$. This extra increase of $T_{e}$ also increases the total energy, resulting in $\tau_{E}{ }^{\text {total }}$ being higher than would be given by the DD scaling relations. These resulting scaling factors were used as inultiplicative factors on the whole, time dependent profiles from 55851, and the resuliting profiles were modeled with TRANSP.

Results from the DT extrapolation simulation and comparisons with the DT equivalent simulation are summarized in Table III. Plots of results are shown in Fig. 5. The peak beam-target contribution is $57 \%$ of the total, and the peak thermonuclear contribution is $24 \%$ of the total. Profiles of the $\alpha$ effects from the extrapolation are quite similar to those of the equivalent simulation, but scaled up in magnitude. The ratio $n_{\alpha}(0) / n_{e}(0)$ increases to $15 \%$ after NBI, as shown in Fig. 5B. Values for $\beta_{\alpha}(0)$ and $\langle\beta \alpha>$ are shown in Fig. $5 \mathrm{C}$. The result for the ratio of the integrated $\alpha$ heating power 
and total thermal losses for the extrapolation, corresponding to the middle curve in Fig. $4 E$, is that $\int P_{\alpha \rightarrow \text { th }} / \int P_{\text {loss }}$ reaches 0.17 near the plasma center.

Since these extrapolations would have to be MHD stable to be viable, the ideal MHD stability has been studied. PEST calculations indicate that the extrapolation discussed here is unstable to high-n modes in the central region. It is not known if minor modifications of the $q_{\psi}$ and pressure profiles would lead to ideal MHD stability. This extrapolation suggests that the stability limits may need to be improved for TFTR to achieve higher $\alpha$ parameters.

Other scenarios for DT experiments which cculd increase the neutron yield and the $\alpha$ parameters have been proposed. They include:

1) Establishing the supershot with a purely $T$ plasma and $T N B I$, then abruptly switching to $\mathrm{D} \mathrm{NBI}$.

2) Using $T$ pellet injection to fuel a target plasma, then using $D \mathrm{NBI}$ and ICRH to heat the plasma.

3) Compressing a supershot.

Scenario 1) would transiently increase the neutron yield, but the NBI sources in TFTR can not be switched from $T$ to $D$ fast enough. Scenario 2) allows comparable values of $Q_{D T}$ and $\beta_{\alpha}$ to be achieved with much less tritium consumption per pulse. Simulations with scenario 3) yielded larger neutron rates and $\beta_{\alpha}$ than those calculated for the DT equivalent simulations, but for short durations.

\section{Theoretical aspects of $\alpha$ instabilities}

There are two classes of collective $\alpha$ instabilities that may have serious consequences for $\alpha$ particle confinement: 1) the toroidicity-induced Alfvén eigenmodes $(T A E)^{37,37,40,41}$ and 2 ) the kinetic ballooning modes $(K B M)^{42}$ instabilities. They are similar in that both are discrete modes, and in that they can be resonantly destabilized by both circulating and trapped $\alpha$ particles when the $\alpha$ pressure is sufficiently steep and $\beta \alpha$ is sufficiently large. The predicted frequencies of these modes are: 


$$
\omega_{T A E} \approx \frac{1}{2} \omega_{\text {Alfvén }}
$$

and

$$
\omega_{\mathrm{KBM}} \approx \omega_{*_{\mathrm{ip}}} \equiv-\mathrm{k}_{\theta} \rho_{\mathrm{i}} v_{\mathrm{ti}} \frac{\mathrm{dln} P_{\mathrm{i}}}{\mathrm{dr}}
$$

(where $\omega_{*_{i p}}$ is the ion diamagnetic drift frequency, $\rho_{i}$ the ion Larmor radius, $P_{i}$, is the ion pressure, and $v_{t i}$ the ion thermal speed), Figures $4 \mathrm{G}$ and $4 \mathrm{H}$ show that the region of steep pressure corresponds roughly to $x \approx r / a<0.4$.

The dominant contribution to instability comes from the most energetic class of $\alpha$ particles that can satisfy the resonance condition, 38,42

$$
\omega \equiv \omega_{d} \pm p \omega_{b, t}, \quad p=0,1,2, . .
$$

where $\omega_{d}$ is the magnetic drift frequency and $\omega_{b(t)}$ is the $\alpha$ bounce (transit) frequency. The frequently quoted criterion that the $\alpha$ particles be super-Alfvénic for TAE modes, however, is not necessary for instability. Generally speaking, the lowest $\beta_{\alpha}$ threshold for these modes occurs when $v_{\alpha}$ Birth $/ v_{\text {Alfvén }} \approx 1$, taking all other parameters to be fixed. Figure $4 \mathrm{~K}$ shows that this condition occurs in the vicinity of $x \approx 0.6$, but this is not the region of steep $\alpha$ pressure gradient (Fig. $4 G$ ). The region $x<0.6$ can still be unstable, but requires higher values of $\beta_{\alpha} .{ }^{40}$

A calculation was performed using the NOVA-K code of the threshold for the TAE instability. 40,41 The plasma parameters were those of 55851 near $3.6 \mathrm{sec}$. The boundary of the unstable region and the trajectory followed by the DT equivalent simulation are shown in Fig. 6 . The trajectory passes close to the boundary around the time of maximum neutron emission, as $\left\langle\beta_{\alpha}\right\rangle$ increases. The boundary of the unstable region changes as the plasma parameters charige, decreasing to lower values of $\langle\beta \alpha\rangle$ as the density and pressure decrease, so when $\langle\beta \alpha\rangle$ is greater than $2 \times 10^{-5}$, mechanisms that would cause $v_{\text {Alfvén }}$ to increase without decreasing $\langle\beta \alpha\rangle$ are predicted to plunge the discharge into the TAE unstable region. This circumstance happens naturally after $\mathrm{NBI}$, and the trajectory is predicted to pass through the unstable region for $0.3 \mathrm{sec}$, offering an opportunity to observe the TAE instability. 
Mechanisms which decrease $\left\langle\beta_{\alpha}\right\rangle$ apparently wouid shift the trajectory below the unstable region. The post-NBI sawteeth sccurring in 55851 were modeled in the DT equivalent, and found to flatten the $n_{\alpha}$ profile briefly, but not to increase the global loss of $\alpha$ particles or to decrease $\left\langle\beta_{\alpha}\right\rangle$, as shown in Fig. $4 \mathrm{~F}$. The trajectory shown in Fig. 6 is typical of the results from other DT equivalent simula:ions of supershots in Table I. The trajectories for the DD fusion products (c.f., Table II) are also indicated in Fig. 6 , and are far below the unstable region.

An important distinction between the TAE and KBM instabilities is that the KBM's are excited only when the plasma is close to the first stability ballooning boundary, whereas TAE modes can exist even below this boundary. The local proximity to ideal ballooning marginality is the relevant criterion. Indeed, the plasma could be close to the ballooning limit in the regions of weak magnetic shear. For the DT equivalent simulation, it can be seen from Fig. $1 \mathrm{~K}$ that in the region $x<0.3$, the plasma is calculated to have low shear. The results in Fig. 2 confirm the conclusion that the discharge is close to the stability limit for the region $0.3<x<0.6$. Since this region overlaps with the region of s.eep $\alpha$ pressure, the destabilization of KBM's appears possible. 35 The threshold value of $\beta_{\alpha}$ at which KBM's become destabilized recuires a careful assessment of the importance of continuum damping for these modes, which awaits further work.

As for the TAE modes, an important question is to determine over what extent of the plasma an Alfvén continuum gap structure exists. 40,41 To leading order in inverse aspect ratio, the center of the gap is rougilly proportional to $\omega_{\text {Alfven }}$, which was plotted in Fig. 4J. To this order, it can be determined on the basis of Fig. $4 \mathrm{~J}$ that a gap structure exists in the region $x<0.4$ since $\omega_{\text {Alfven }}$ is relatively flat here. However, inverse aspect ratio corrections will enhance the gap center values, so that the gap structure may extend across the plasma to the edge. 41 This is the most unstable scenario, and theoretical understanding in this area is still evolving.

\section{Summary}

The TRANSP code has been used to model pairs of DT / DD discharges in TFTR. The DD discharges are actual TFTR discharges, including supershots with the highest measured neutron rates and $Q_{D D}$. Detailed comparisons of predictions and measurements are shown for one supershot to indicate that accurate agreement with 
measurements is achieved. Corrections, such as for the $T_{i}-T_{i m p}$ differences were included and found to change the calculated neutron rates by $\approx 5 \%$. Fusion products from the $D D$ reactions in this discharge are calculated and, as expected, their parameters are very small.

The DT equivalent simulations are conservative, assuming minimal alierations of actual $D D$ discharges. These calculations give the computed ratio, $Q_{D T} / Q_{D D}$ for supershots to be typically in the range $150-175$, with the maximum calculated QDT $\approx$ 0.3. The maximum $\alpha$ parameters are $\beta_{\alpha}(0) \approx 0.5 \%$ and $n_{\alpha}(0) / n_{e}(0) \approx 0.35 \%$. The $\alpha$ effects appear large enough that the toroidal Alfven eigenmode and kinetic ballooning modes might be observed, particularly during the density decreasing period after the end of NBI. The sawteeth often observed after NBI in supershots are predicted to not decrease $\left\langle\beta_{\alpha}\right\rangle$, and thus to not terminate the TAE instability.

A DT extrapolation of a MHD-free supershot to full NBI parameters is derived from empirical supershot scaling relations. The value of $Q_{D T}$ is calculated to increase from 0.28 at $25 \mathrm{MW} \mathrm{NBI}$ to 0.37 at $35 \mathrm{MW} \mathrm{NBI}$. The MHD stability of this extrapolation is, however problematic. 


\section{Acknowledgements}

This research was supported by the U.S. Department of Energy Contract No. DE-ACO2-76-CHO-3073. 


\section{Appendix A}

The impurity temperature $T_{i m p} \equiv T_{X}$ is calculated in TRANSP, using the local heating power density delivered to impurities (including beam heating, RF heating, compressional heating, viscous heating, and fast ion heating from fusion products), power flows due to particle convection, and a local model of conductive losses.

The equations that describe the time evolution of impurity energy density and hydrogenic energy density are:

$$
\begin{aligned}
& \frac{\partial}{\partial t}\left(\frac{3}{2} n_{x} T_{x}\right)=q_{x}-c \nabla \cdot\left(\Gamma_{x} T_{x}\right)+\frac{\frac{3}{2} n_{x}\left(T_{i}-T_{x}\right)}{\tau_{x i}}+\frac{\frac{3}{2} n_{x}\left(T_{e}-T_{x}\right)}{\tau_{x e}}-\frac{\frac{3}{2} n_{x} T_{x}}{\tau_{E}{ }^{i o n}} \\
& \frac{\partial}{\partial t}\left(\frac{3}{2} n_{i} T_{i}\right)=q_{i}-c \nabla \cdot\left(\Gamma_{i} T_{i}\right)+\frac{\frac{3}{2} n_{i}\left(T_{x}-T_{i}\right)}{\tau_{i x}}+\frac{\frac{3}{2} n_{i}\left(T_{e}-T_{i}\right)}{\tau_{i e}}-\frac{\frac{3}{2} n_{i} T_{i}}{\tau_{E}{ }^{i o n}}
\end{aligned}
$$

where subscripts $x$ and $i$ indicate impurity and main ion, respectively, $q$ is the total heating power density, $\Gamma$ is the particle flux, derived from the continuity equation for each species, $c$ is the convective multiplier (supplied by the user), $\tau_{x i}$ is the temperature relaxation time for impurities on the main ions, $\tau_{i x}$ is the temperature relaxation time for the main ions on impurity ions, $\tau_{x e}$ is the temperature relaxation time of impurities on electrons, $\tau_{i x}$ is the temperature relaxation time of main ions on electrons, and $\tau_{E}{ }^{i o n}$ is a local ion energy confinement time whose value will be determined self-consistently.

To determine the proper expression for $\tau_{E}$ ion which makes Eqs. (A1) and (A2), a self-consistent local model of the ion energy balance, we will sum the equations, making use of the following identities and definitions: 


$$
\begin{aligned}
& 0=\frac{\frac{3}{2} n_{x}\left(T_{x}-T_{i}\right)}{\tau_{x i}}+\frac{\frac{3}{2} n_{i}\left(T_{i}-T_{x}\right)}{\tau_{i x}} \\
& q_{e i} \equiv \frac{\frac{3}{2} n_{x}\left(T_{e}-T_{x}\right)}{\tau_{x e}}+\frac{\frac{3}{2} n_{i}\left(T_{e}-T_{i}\right)}{\tau_{i e}} \\
& q_{h} \int q_{i}+q_{x}+q_{b t h} \\
& q_{\text {conv }} \equiv c \nabla \cdot\left(\Gamma_{x} T_{x}+\Gamma_{i} T_{i}\right) \\
& W_{i} \int \frac{3}{2}\left(n_{i} T_{i}+n_{x} T_{x}\right)
\end{aligned}
$$

where qei represents the total power density transfer from electrons to all thermal ions (hydrogenic + impurity), $q_{\mathrm{h}}$ is the total heating power density to the thermal ions, $q_{\mathrm{bth}}$ is the power carried by the thermalizing beam ions $\left(\frac{3}{2} s_{b}{ }^{\text {th }} T_{i}\right), q_{c o n v}$ is the total convective power flow, and $W_{i}$ is the total thermal ion energy density. The sum of Eqs. (A1) and (A2) becomes

$$
\frac{\partial W_{i}}{\partial t}=q_{\mathrm{h}}+q_{\theta i}-q_{\mathrm{conv}}-\frac{W_{\mathrm{i}}}{\tau_{E^{i o n}}}
$$

Since all of the terms in this equation are available to TRANSP except $\tau_{E}$ ion, we can solve for it:

$$
\tau_{E}{ }^{i o n}=\frac{W_{i}}{q_{h}+q_{e i}-q_{\text {Conv }}-\frac{\partial W_{i}}{\partial t}}
$$

For each transport tim $\rightarrow$ step, TRANSP advances Eq. (A1) in time, using the $\tau_{E^{i o n}}$ determined from Eq. (A5). 


\section{References}

1D. L. Jassby, C. W. Barnes, M. G. Be!l, et al,, Physics of Fluids, B3, 2308 (1991), and references therein.

2D. M. Meade, V. Arunasalam, C. W. Barnes, et al., in Proceedings of the Thirteenth International Conference on Plasma Physics and Controlled Nuclear Fusion Research, (Washington DC, Oct 1991) Paper IAEA-CN-53/A-1-1.

${ }^{3} \mathrm{M}$. Redi, S. Zweben, and G. Bateman, Fusion Technol. 1357 (1988).

4S. J. Zweben, H. P. Furth, D. R. Mikkelsen, et al., Nucl. Fusion 28 ?230 (1988).

5J. D. Strachan, S. J. Zweben, C. W. Barnes, et al., in Proceedings of the Tweltth International Conference on Plasma Physics and Controlled Nuclear Fusion Research, Nice, France, (IAEA, Vienna, 1989), 1, p 257.

6R. J. Goldston, in Basic Physical Processes of Toroidal Fusion Plasmas (Proc. Course and Workshop Varenna, 11085) EUR-10418-EN, (CEC, Brussels, 1986) 1, p. 165.

${ }^{7}$ R. J. Hawryluk, "An Empirical Approach to Tokamak Transport", in Physics of Plasmas Clnse to Thermonuclear Conditions, ed. by B. Coppi, et al., (CEC, Brussels, 1980), Vol. 1, p. 110.

8M. C. Zarnstorff, K. McGuire, M. G. Bell, et al., Phys. Fluids B2 (1990) 1852.

9J. D. Strachan, M. Bitter, A. T. Ramsey, et al., Phys. Rev. Lett., 58, 1004 (1987). ${ }^{10}$ B.B. Kadomtsev, Sov. J. Plasma Phys. 1 (1975) 389.

$11 \mathrm{C}$. Mercier and $\mathrm{H}$. Luc, "The MHD Approach to the Problem of $F$ asma Confinement in Closed Magnetic Configurations", EUR 5127e, Commission of the European Communities, Luxembourg, (1974).

12The JET Team, Plasma Phys. Controlled Fusion, 32, 1083, (1990).

13B. Balet, J. G. Cordey and P. M. Stubberfield, Plasma Phys. Controlled Fusion, 32, 106, (1990).

14J. A. Snipes, J. L. Terry, E. S. Marmar, K. W. Hill, et al., in Proceedings of the 18th European Conference on Controlled Fusion and Plasma Physics, Ill 141 (June 1991). 15G. Taylor, P. Efthimion, M. McCarthy, et al., Rev. Sci. Instrum. 55, 1739 (1984).

${ }^{16}$ A. Cavallo, R. C. Cutler, and M. P. McCarthy, Rev. Sci. Instrum. 59, 899 (1988). 17F. J. Stauffer, D. A. Boyd, R. C. Cutler, et al., Rev. Sci. Instrum. 59, 2139 (1988). 18D. Johnson, N. Bretz, B. Grek, D. Long, R. Pallandino, and E. Tolnas, Rev. Sci. Instrum. 57, 1856 (1986).

19B. Stratton, R. J. Fonck, K. P. Jaehnig, et al., in "The Proceedings of the IAEA Technical Committee Meeting on Time Resolved Two- and Three-Dimensional Plasma Diagnostics", Nagoya, Japan, Nov, 1990. 
20H. Hsuan, M. Bitter, J. E. Rice, et al., Rev. Sci. Instrum., 59, 2127 (1988).

21H. Park, Plasma Phys. Controlled Fusion, 31, 2035 (1989).

22M. C. Zarnstorft, M. G. Bell, M. Bitter, et al., Phys. Rev. Lett. 60, 1306 (1988).

${ }^{23}$ R.J. Fonck, A.T. Ramsey, R.V. Yelle, Appl. Optics, 21, 2115-2123 (1982).

24K. W. Hill, M. Bitter, N. L. Bretz, et al., Nucl. Fusion 26, 1131, (1986).

25R. Budny and the TFTR group, J. Nucl. Mat. 176 \& 177, 427 (1990).

26H. Hendel, R. W. Pallandino, C. W. Barnes, et al., Rev. Sci. Instrum. 61, 1900 (1990).

27H. H. Towner and R. Goldston, Bull. Am. Phys. Soc. 29, 1305 (1984).

${ }^{28}$ B. H. Duane, "Fusion Cross Section Theory", in W. C. Wolkenhauer, editor, Annual report on CTR Technology 1972. Batelle Pacific Northwest Laboratory, Richland, WS, 1972. BNWL-1685.

29H.-S. Bosch, IPP 1/252 (Sept 1990).

30) A. L. Roquemore, R. C. Chouinard, M. Diesso, R. Palladino, J. D. Strachan, and G.

D. Tait., Rev. Sci. Instrum. 61, 3163 (1990).

31K. W. Hill, S. von Goeler, M. Bitter, et al., Rev. Sci. Instrum. 56, 830 (1985).

32L. C. Johnson, M. Bitter, R. Chouinard, and K. M. Young, Rev. Sci. Instrum. 57, 2133 (1986).

33J. Manickam, V. Arunasalam, C. W. Barnes, et al., Twelfth International Conference on Plasma Physics and Controlled Nuclear Fusion Research, Nice, France, October 1988.

34J. Delucia, S. C. Jardin, and A. M. M. Todd, J. Comp. Phys. 37, 183-204 (1980).

${ }^{35}$ M. W. Phillips, A. M. Todd, M. H. Hughes, J. Manickam, J. L. Johnson, R. R. Parker, Nucl. Fusion 28, 1499 (1988).

36R. C. Grimm, R. L. Dewer, and J. Manickam, J. Comput. Phys. 49, 94-117 (1983).

37L. Chen, in "Theory of Fusion Plasmas", Ed. J. Vaclavik, F. Troyon, and E. Sindoni (CRPP, Association EURATOM, 1988), p. 327.

$38 \mathrm{H}$. Biglan, F. Zonca, and L. Chen, PPPL-2789, submitied to Phys. Rev. Lett.

79.J. D. Strachan, Bulletin of the Am. Phys. Soc., Nov., 1991, and to be published.

${ }^{40} \mathrm{C}$. Z. Cheng, Princeton Plasma Physics Laboratory Report PPPL-2717, October, 1990, to be published in Phys. Fluids B, 1991.

${ }^{41} \mathrm{C}$. Z. Cheng, Proceedings of the First TFTR DT workshop (Princeton, NJ, March, 1990).

${ }^{42} \mathrm{H}$. Biglari and L. Chen, Princeton Plasma Physics Laboratory Report PPPL-2790, submitted to Phys. Rev. Lett. 
Table I

TFTR discharges used for DT equivalent simulations, and summary of peak results.

Measured TRANSP results

Discharge type Ip $\quad P_{\text {NB }} \quad x_{i} \quad Q_{D D}$ MA MW $\left(\times 10^{3}\right)$ $Q_{D T} / Q_{D D} \quad$ ratio $\quad \beta_{\alpha}(0)<\beta_{\alpha}>$ $\% \quad\left(x 10^{4}\right)$

\begin{tabular}{lllllllllll}
\hline 26627 & supershot & 0.85 & 10.6 & $2 \chi_{e}$ & 0.91 & $0.206 / 0.00103$ & 201 & 0.10 & 1.1 \\
35782 & supershot & 1.4 & 22.0 & CHERS & 1.62 & $0.297 / 0.00181$ & 164 & 0.28 & 4.2 \\
37081 & supershot & 1.6 & 30.4 & CHERS & 1.50 & $0.234 / 0.00154$ & 152 & 0.28 & 3.2 \\
37083 & supershot & 1.6 & 30.2 & CHERS & 1.36 & $0.239 / 0.00147$ & 163 & 0.25 & 3.1 \\
37084 & supershot & 1.6 & 30.2 & CHERS & 1.47 & $0.246 / 0.00152$ & 162 & 0.20 & 3.1 \\
37085 & supershot & 1.6 & 25.0 & CHERS & 1.59 & $0.292 / 0.00181$ & 161 & 0.28 & 2.9 \\
$45950 \mathrm{a}$ & L-mode & 2.0 & 11.4 & CHERS & 0.41 & $0.098 / 0.00041$ & 239 & 0.03 & 0.4 \\
$47394 \mathrm{~b}$ & supershot & 1.6 & 24.3 & $1 \chi_{e}$ & 1.83 & $0.255 / 0.00168$ & 152 & 0.02 & 2.7 \\
$53793 \mathrm{C}$ & supershot & 1.7 & 26.2 & $2 \chi_{e}$ & 1.43 & $0.258 / 0.00171$ & 151 & 0.30 & 4.6 \\
$53848 \mathrm{C}$ & supershot & 1.6 & 25.5 & $2 \chi_{e}$ & 1.74 & $0.291 / 0.00183$ & 159 & 0.55 & 6.0 \\
55053 & pellets & 2.0 & 22.8 & CHERS & 0.84 & $0.124 / 0.00103$ & 120 & 0.14 & 1.4 \\
55804 & supershot & 1.84 & 32.5 & $2 \chi_{e}$ & 1.66 & $0.291 / 0.00196$ & 165 & 0.32 & 4.3 \\
55806 & supershot & 1.6 & 32.5 & CHERS & 1.81 & $0.298 / 0.00188$ & 156 & 0.33 & 4.7 \\
55851 & supershot & 1.6 & 24.6 & CHERS & 1.81 & $0.275 / 0.00159$ & 173 & 0.33 & 3.9
\end{tabular}

asawteeth during NBI

bended with a disruption and poorly diagnosed, so the modeling is suspect

cquoted $Q$ values occur just before the mild compression in major and minor radius, and the quoted $\beta_{\alpha}(0)$ and $<\beta_{\alpha}>$ values occur during the compression 


\section{Table II}

Fusion products (fp) parameters computed for the DD reactions in 55851.

Fusion Product

${ }^{3} \mathrm{He}$

T

$n_{f p}(0) / n_{e}(0)$

$3.2 \times 10^{-5}$

$8.5 \times 10^{-6}$

$\beta_{f p}(0)$

$1.3 \times 10^{-5}$

$4.4 \times 10^{-6}$

$<\beta_{f p}>$

$9.0 \times 10^{-7}$

$8.5 \times 10^{-7}$

$v_{f p}$ Birth $/ v_{\text {Alfvén }}(0)$

0.90

1.05 


\section{Table III}

Summary of parameters and results from the DT equivalent simulation of 55851 and from the DT extrapolation based on empirical scaling relations and improved stability limits.

\section{DTequivalent}

PNBI (MW)

IP (MA)

$\mathrm{n}_{\mathrm{e}}(0)\left(10^{\left.20 / \mathrm{m}^{3}\right)}\right.$

$\mathrm{T}_{e}(0)(\mathrm{KeV})$

$\mathrm{T}_{\mathrm{i}}(0)(\mathrm{KeV})$

$Z_{\text {eff }}$

$\beta_{\text {norm }}$

QDT

$\beta_{\alpha}(0) \%$

$<\beta_{\alpha}>\%$

$n_{\alpha}(0) / n_{e}(0) \%$

$\tau_{\mathrm{E}}^{\text {thermal }}$ (sec)

$\tau \mathrm{E}^{\text {total }}$ (sec)

$\int P_{\alpha->e} d V(M W)$

$v_{\alpha}{ }^{\text {Birth } / v_{\text {Alfvén }}(0)}$
24.6

1.58

0.95

10.2

22.5

2.8

2.25

0.28

0.28

0.04

0.2

0.13

0.17

1.1

1.85

\section{DT extrapolation}

35

1.95

1.07

12.8

24.5

2.3

2.8

0.37

0.7

0.09

0.6

0.10

0.19

2.1

2.0 


\section{Figures}

Fig. 1. TRANSP results and measurements for 55851 .

A) Components of the plasma current versus time. The total current was measured, and input into TRANSP as a boundary condition. The beam driven and bootstrap currents are calculated in TRANSP from the beam deposition and total pressure profiles. The relative amounts of these non-inductive currents are typical of those calculated for supershots.

B) Central values of the temperatures versus time. $T_{e}$ is computed in TRANSP

by symmetrizing the smoothed time-dependent second harmonic ECE, which was cross-calibrated by the Thomson scattering profile at $3.46 \mathrm{sec}$. Timp is fit by TRANSP using the time-dependant CHERS measurements. The effects of sawteeth in the pre- and post-NBI plasma are shown.

C) Temperature profiles and measurements at the TVTS time. The TVTS profile is shown. $T_{e}$ is derived from ECE, measured by GPC, cross-calibrated to TVTS, and symmetrized by in/out averaging. Timp is fit by TRANSP using the CHERS measurements from the magnetic axis out to larger major radii. The $T_{\text {imp }}$ profile measured from Doopler broadening of iron $X$-ray lines is shown for comparison. Error bars for the measurements are shown.

D) Density profiles and measurements at the TVTS time. The TVTS profile is scaled in magnitude so that the peak density is that measured by MIRI. The TRANSP $n_{e}$ profile is calculated by symmetrizing the smoorhed MIRI profile. The thermal deuterium density, $n_{D}$ and beam density, $n_{\text {beam }}$ are calculated in TRANSP.

E) Surface voltage computed in TRANSP using neo-classical resistivity, beamdriven, and bootstrap currents, and compared with measured values. The errors on the measured values are less than \pm 0.04 Volts during quiescent periods (when $\mathrm{dE} / \mathrm{dt}$ is small).

F) $Z_{\text {eff }}$ computed from the measured visible bremsstrahlung, and $Z_{\text {met }}$ (the contribution from $\mathrm{Cr}, \mathrm{Fe}$, and $\mathrm{Ni}$ to $\mathrm{Z}_{\text {eff, }}$, measured by PHA), versus time.

$G)$ Comparison of the predicted and measured perpendicular energy. The experimental errors for the measurement are $\pm 150 \mathrm{KJ}$.

H) Total neutron emission rate compared with the measured value and the c. sulated 
beam-target, thermonuclear, and beam-beam components versus time. The absolute error bars for the measurement are $\pm 14 \%$. Results from a steady state calculation using SNAP are shown.

1) Comparison of the simulated and measured neutron emission along vertical chords. The experimental errors are estimated to be $\pm 20 \%$ near the peak, and larger in the inner and outer edges where the measurement is comparable to the scattered signal.

J) Computed location of the magnetic axis compared with the location of the region of

maximum soft $X$-ray emission. The errors become larger after the NBI.

K) Profiles of $q_{\psi}$ and shear. The profiles do not vary appreciably between 3.5 and $3.9 \mathrm{sec}$.

L) Profiles of the thermal and total pressure.

Fig. 2. Profile of the gradient of the total pressure with respect to the poloidal flux versus the square root of the normalized poloidal flux, compared with the boundary of the high-n ballooning unstable region.

Fig. 3. Projected $Q_{D T}$ calculated from the predicted ratio $Q_{D T} / Q_{D D}$ times the measured $Q_{D D}$ versus the measured $Q_{D D}$ for the DD/DT equivalent pairs in Table I.

Fig. 4. Results from the DT equivalent simulation based on 55851 .

A) DT fusion reaction rates versus time.

B) Profile of the DT neutron emissivities.

C) Measured profile of $n_{e}$, and the computed profiles of nbeam, $n_{D}, n_{T}, n_{i m p}$ and $n_{\alpha}$ at the end of the NBI.

D) Volume-integrated heating powers versus time.

E) Ratio of the volume-integrated $\alpha$ heating power to the volume-integrated power loss rate for the thermal ions, electrons, and both at the end of NBI.

F) Components of $\beta(0)$ and $\left\langle\beta_{\alpha}\right\rangle$ versus time. Effects of post-NBI sawteeth can be seen in $\beta_{\alpha}(0)$.

G) Profiles for components of $\beta_{\text {tor. }}$.

H) Profile of the $\alpha$ production, density, heating, and $\beta_{\alpha}$ normalized to 1 at $x=0$.

1) Profiles of the $\alpha$ slowing down time and the average $\alpha$ energy. 
J) Profiles of the Alfvén frequency and the Alfven speed. The speed of the initial $\alpha$ particles is indicated.

K) Profile of the ratio of the $\alpha$ birth speed and the Alfven speed.

Fig. 5. TRANSP results for the DT extrapolation.
A) Profile of the ratio of the volume integral of the $\alpha$ heating of electrons and the beam and ion-electron coupling.
B) Ratio of the central densities of $\alpha$ and electrons.
C) Central and volume averaged $\beta_{\alpha}$. Sawteeth were not modeled.

Fig. 6. Calculated boundary of the TAE instability region in volume-averaged $\beta$ fusion product vs the central value of the ratio of the fusion product birth speed and the Alfven speed. The $\beta$ fusion product was assumed to decay decay length in the DT equivalent simulation lies between these values. The trajectory of the DT $\rightarrow \alpha$ equivalent simulation is indicated, with the closed circle indicating the end of NBI. The trajectories of the $D D \rightarrow{ }^{3} \mathrm{He}$ and $\mathrm{DD} \rightarrow T$ predictions are are also shown. 


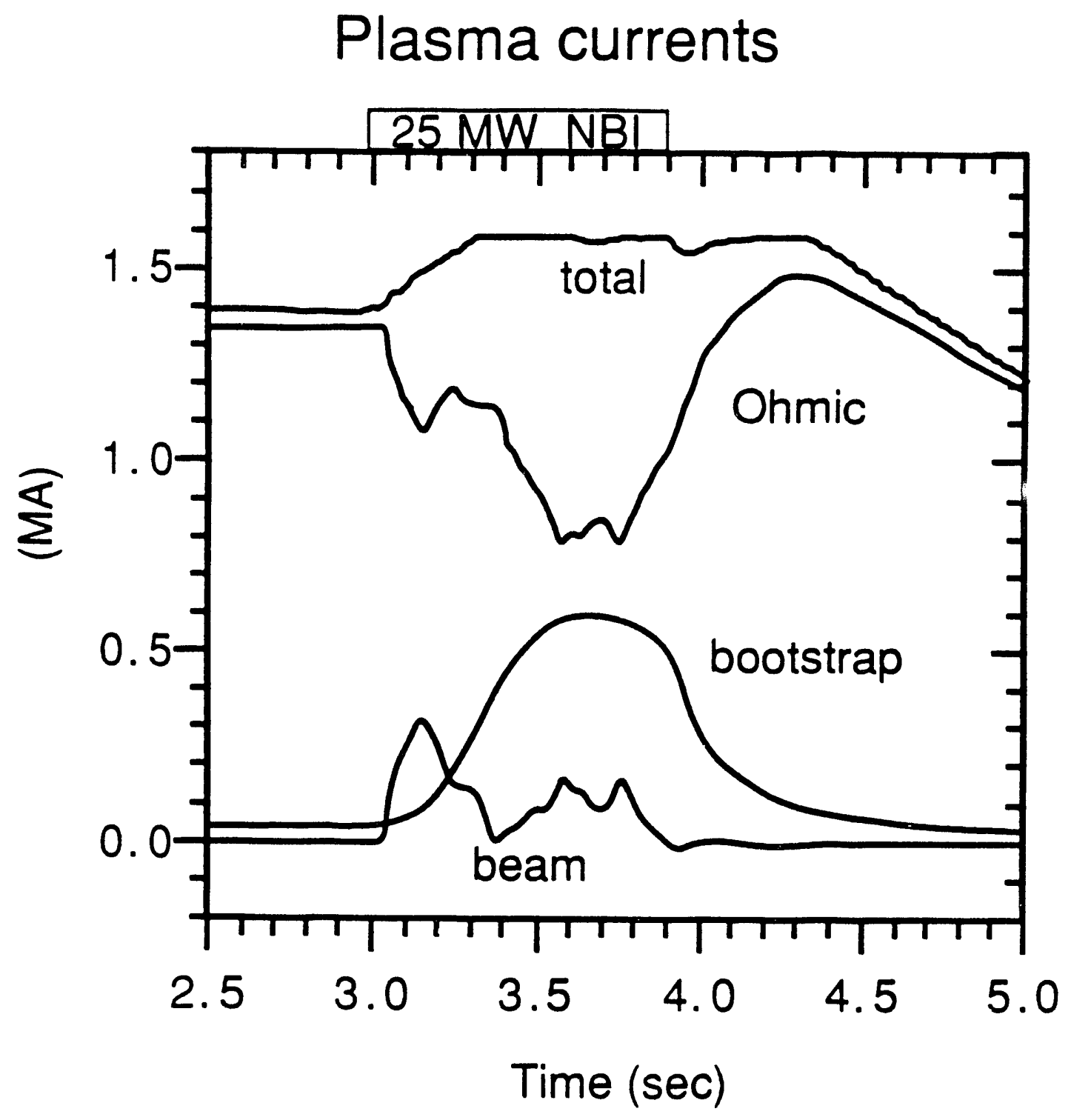

Fig $1 \mathrm{~A}$ 


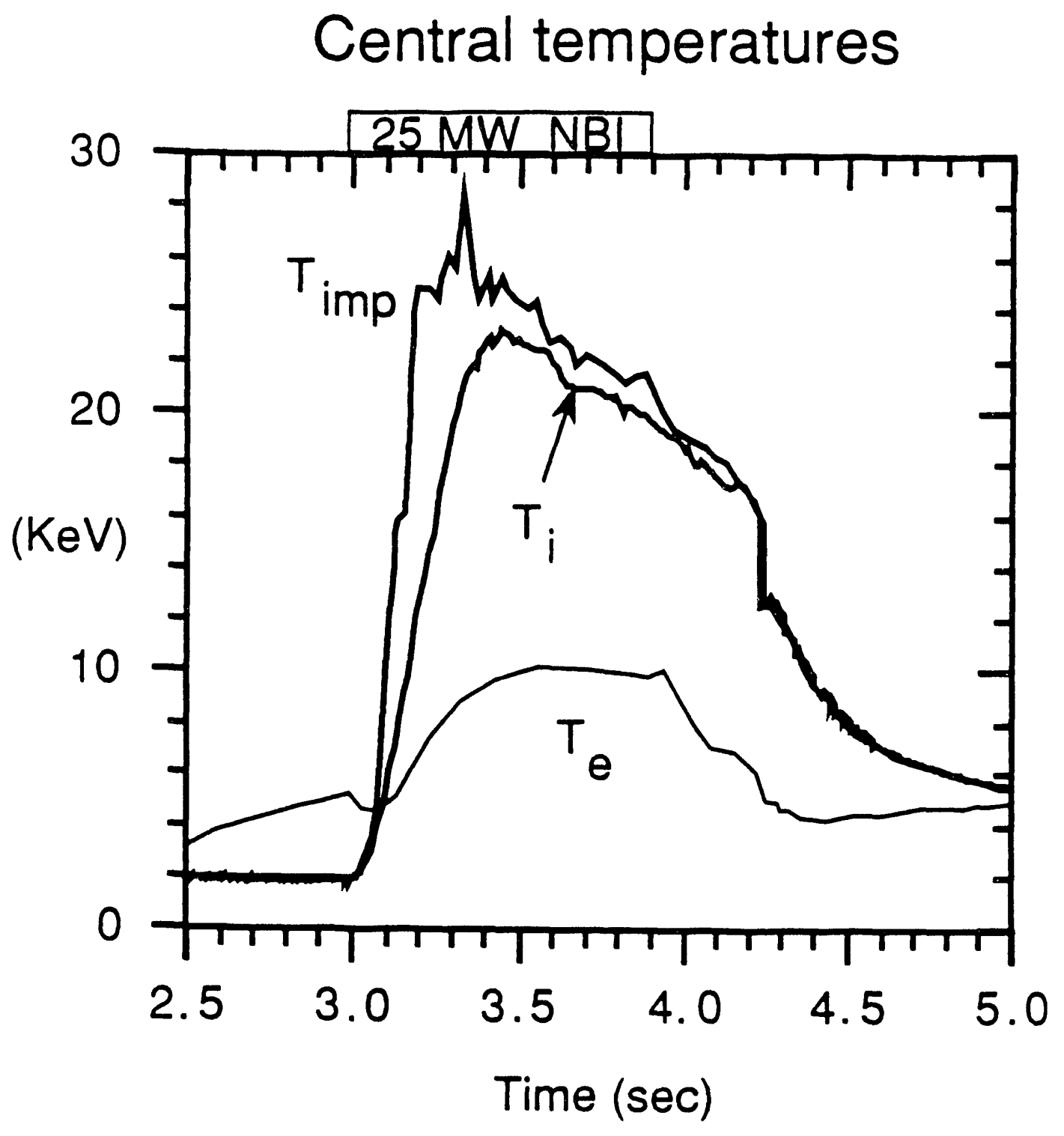

Fig 1B 
Temperature profiles

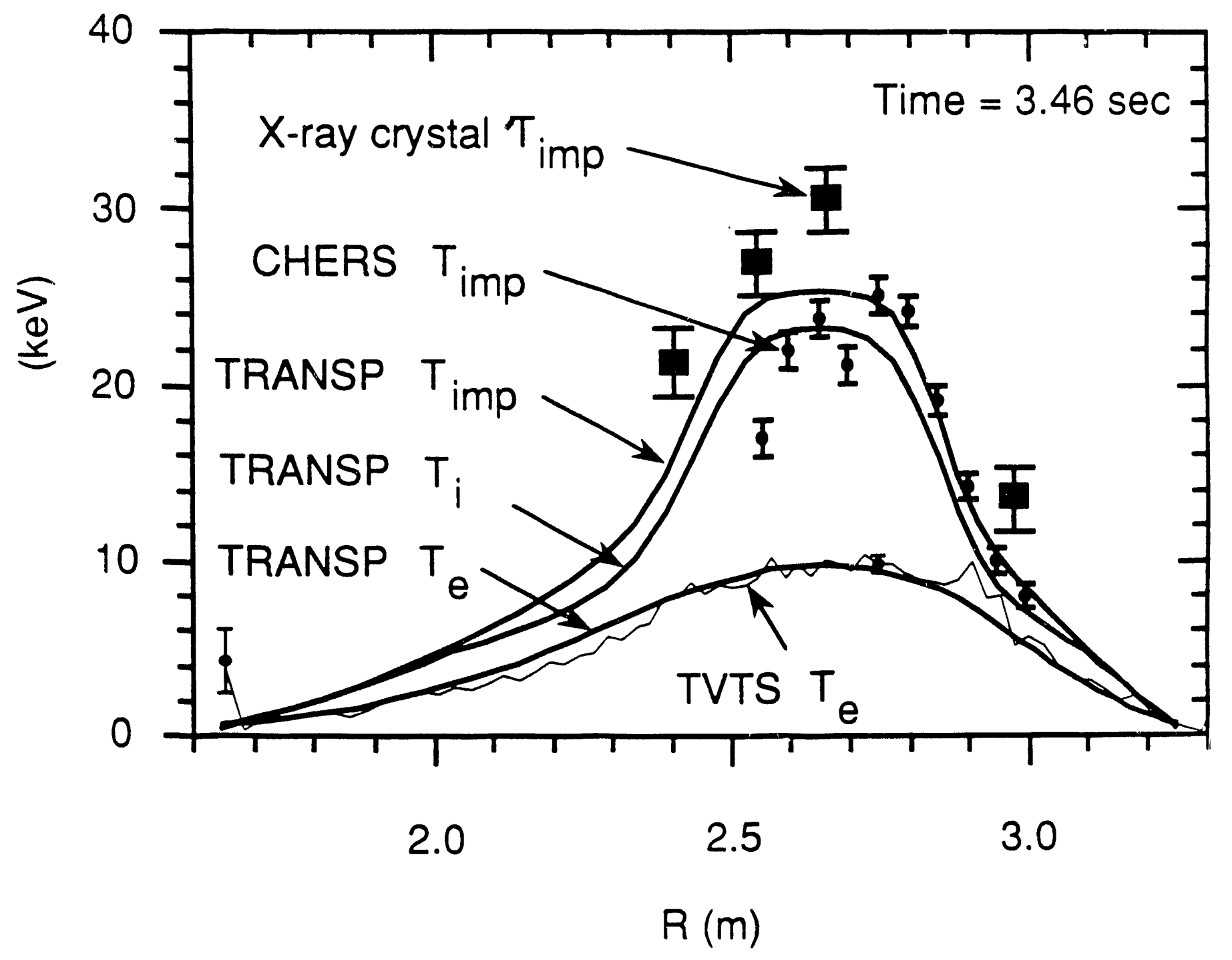

Fig $1 C$ 
Density profiles

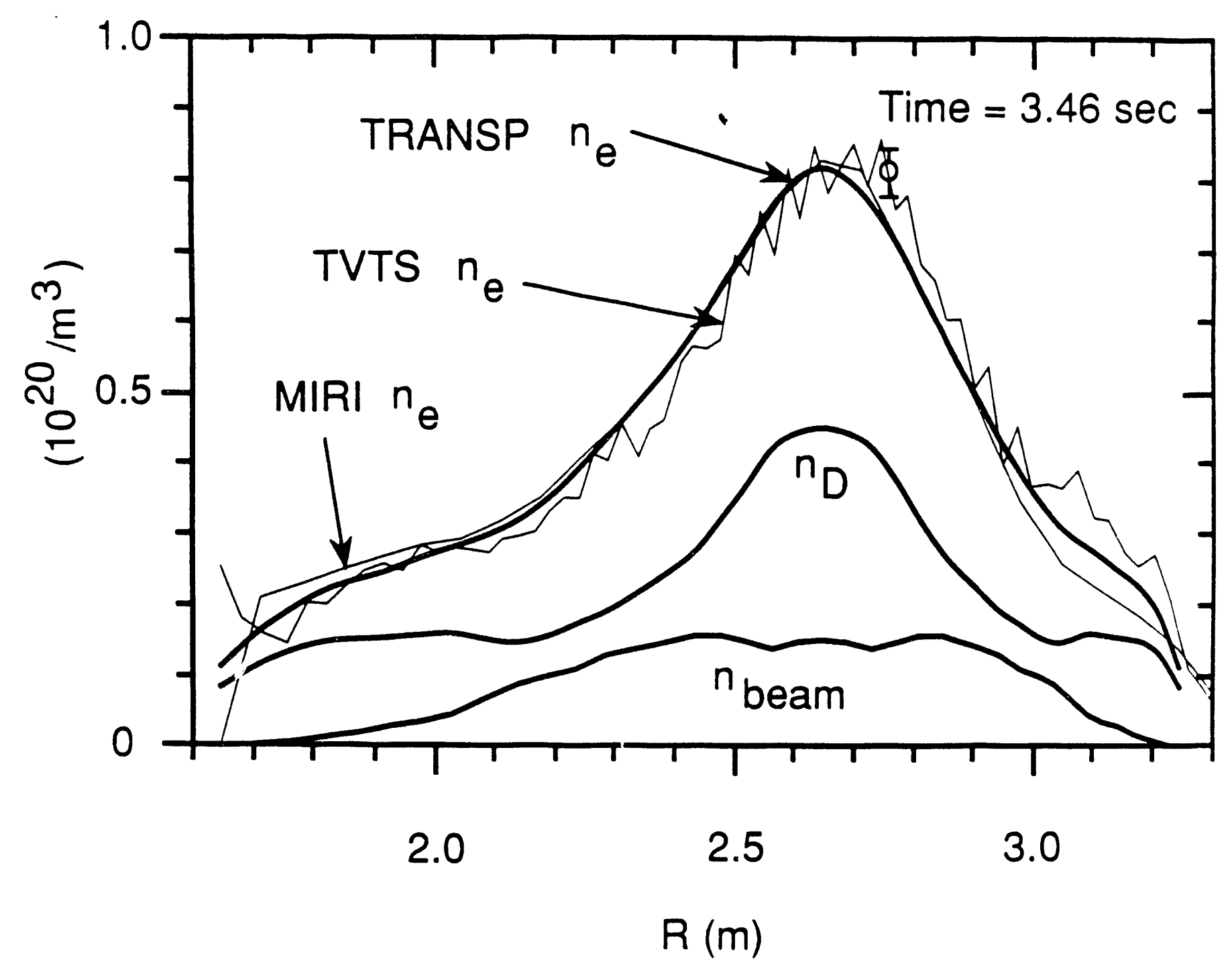

Fig 1D 


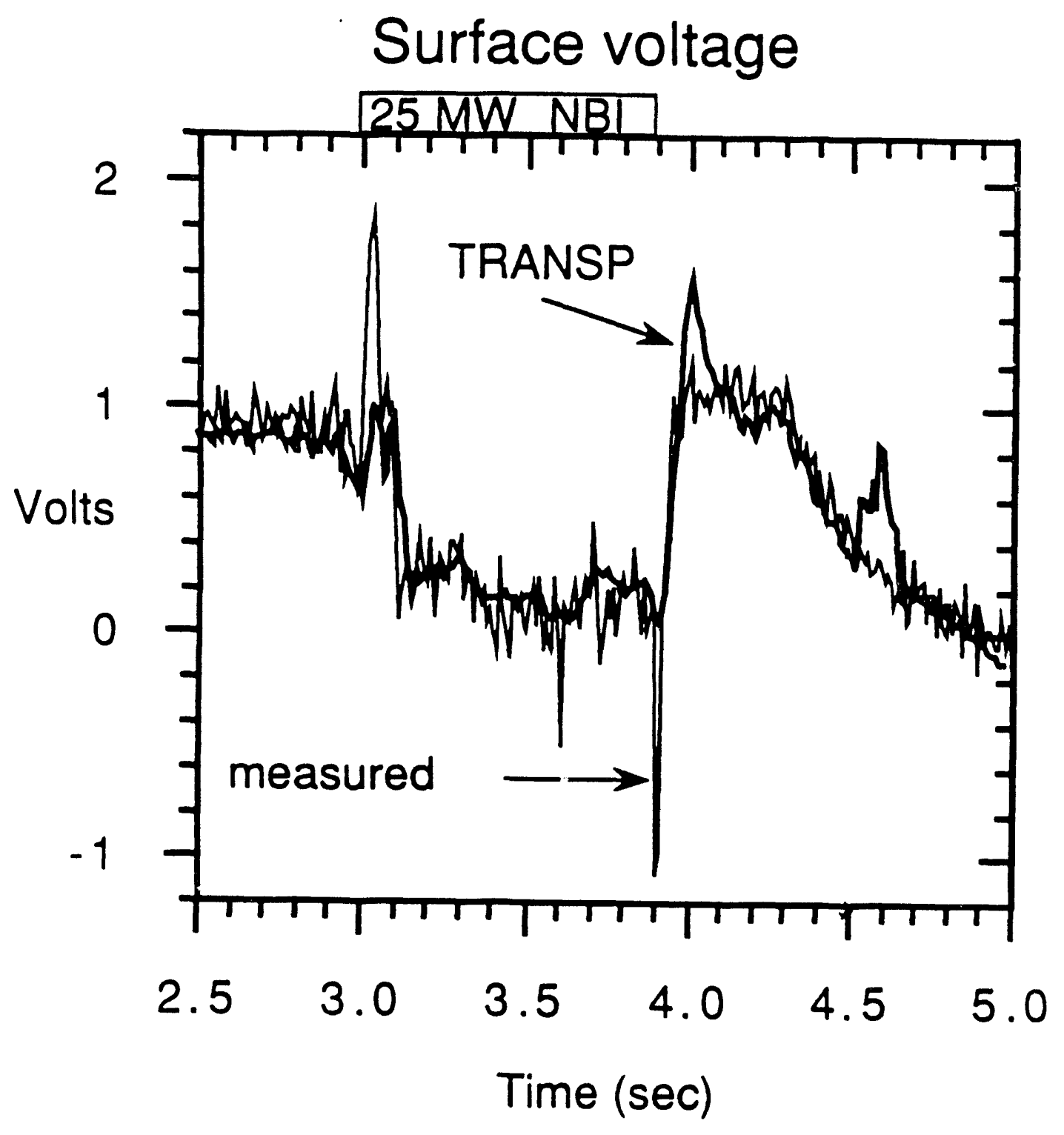

Fig 1E 


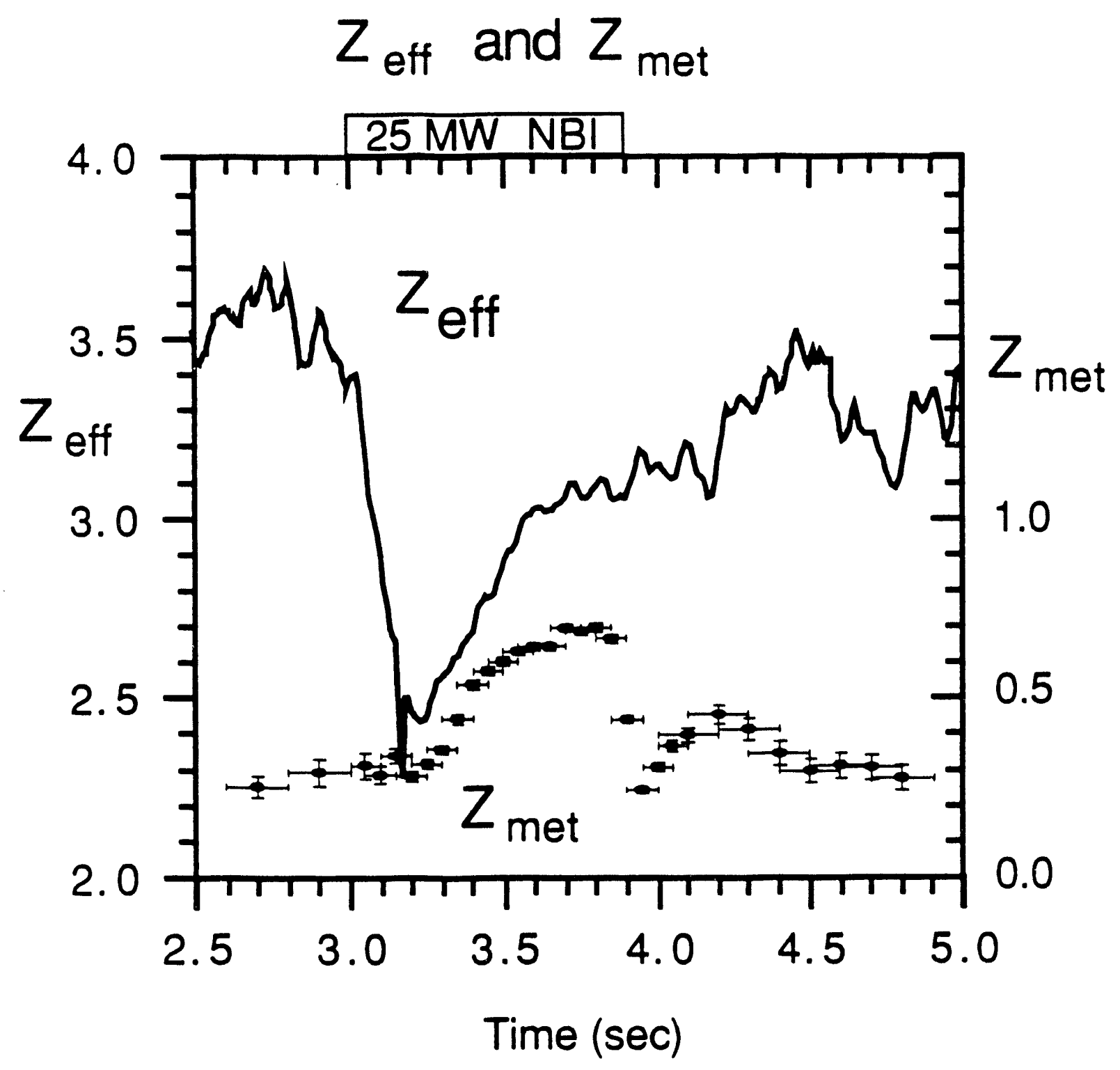

Fig $1 F$ 


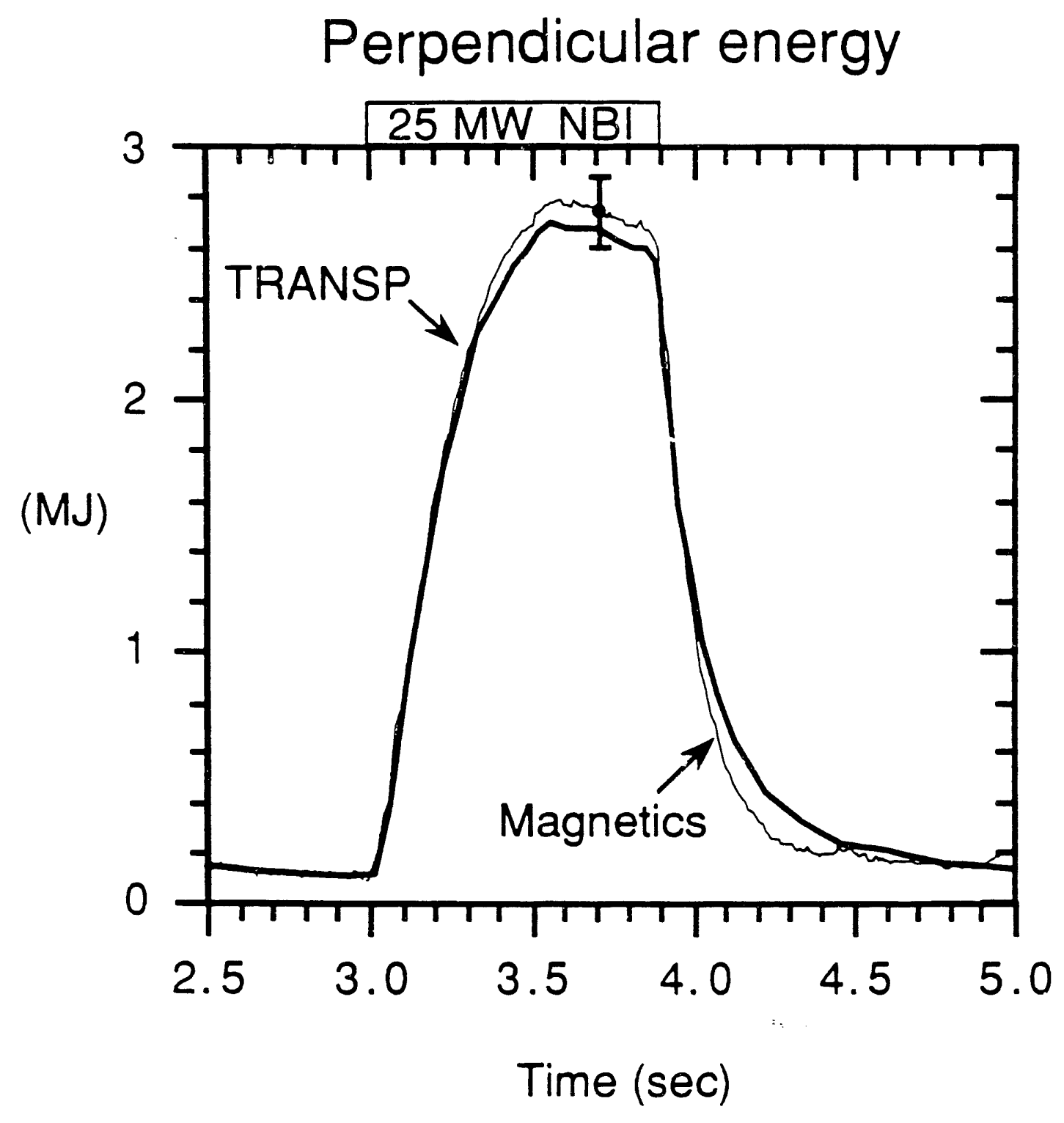

Fig $1 G$ 


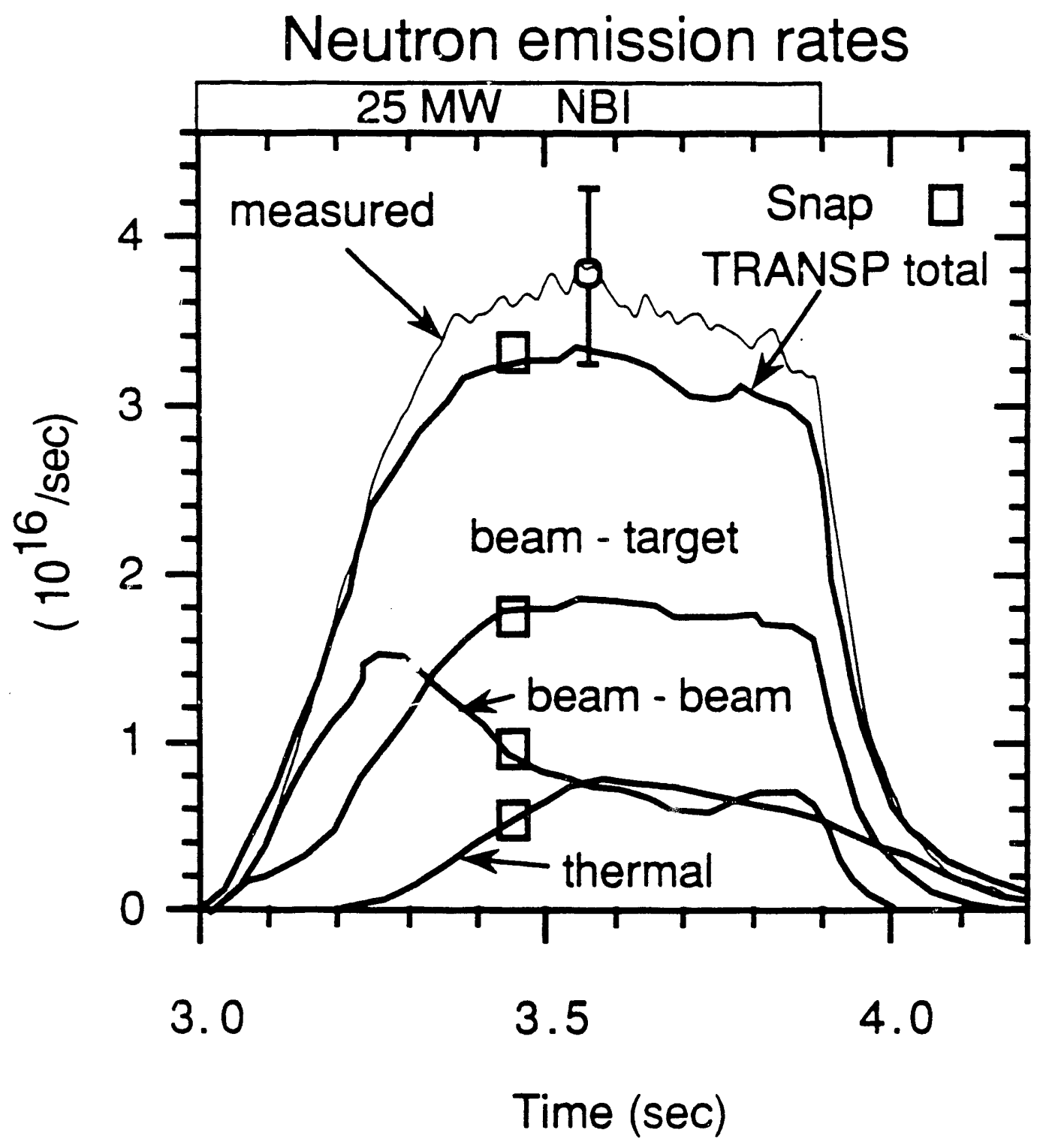

Fig $1 \mathrm{H}$ 


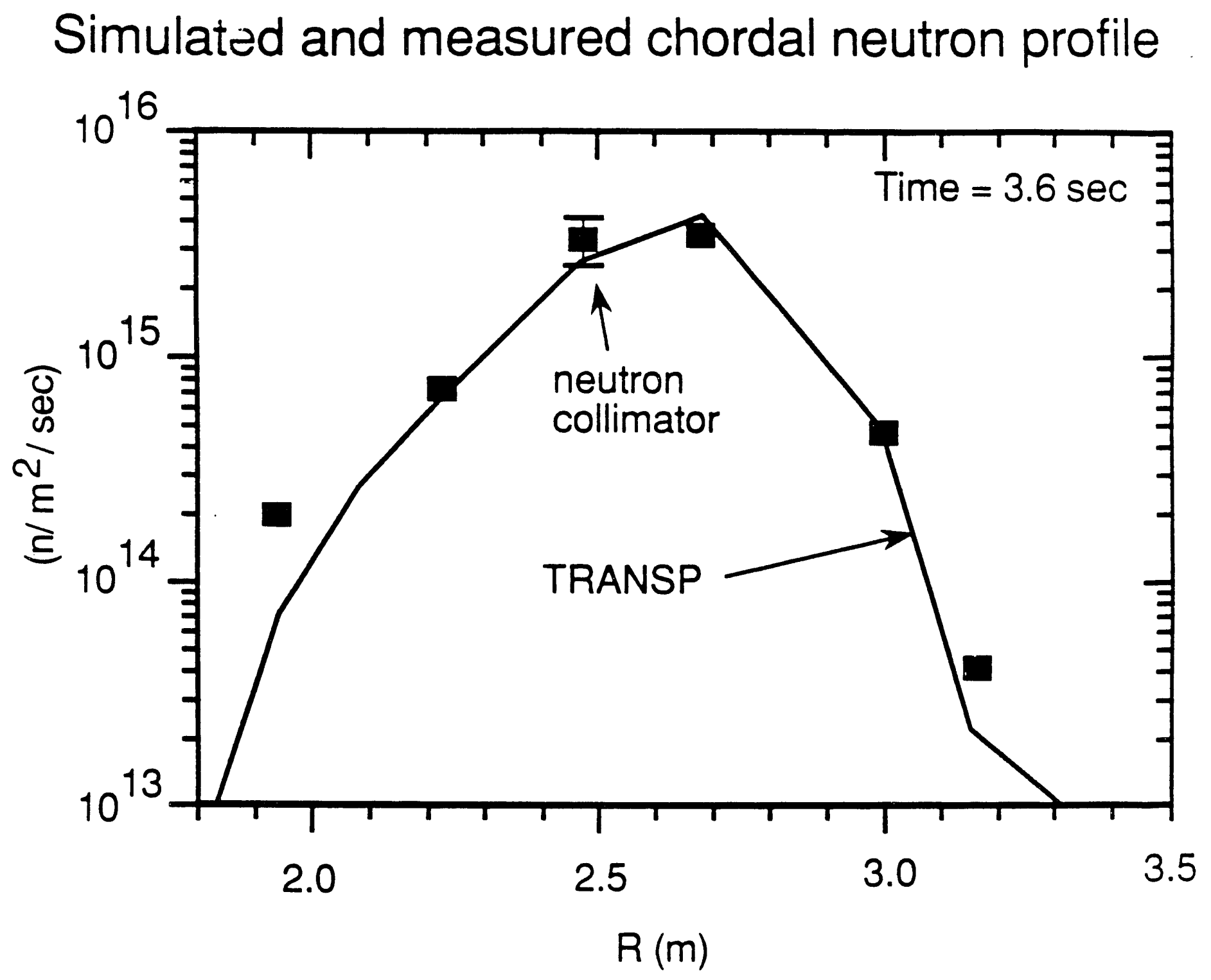

Fig 11 
Radius of the magnetic axis

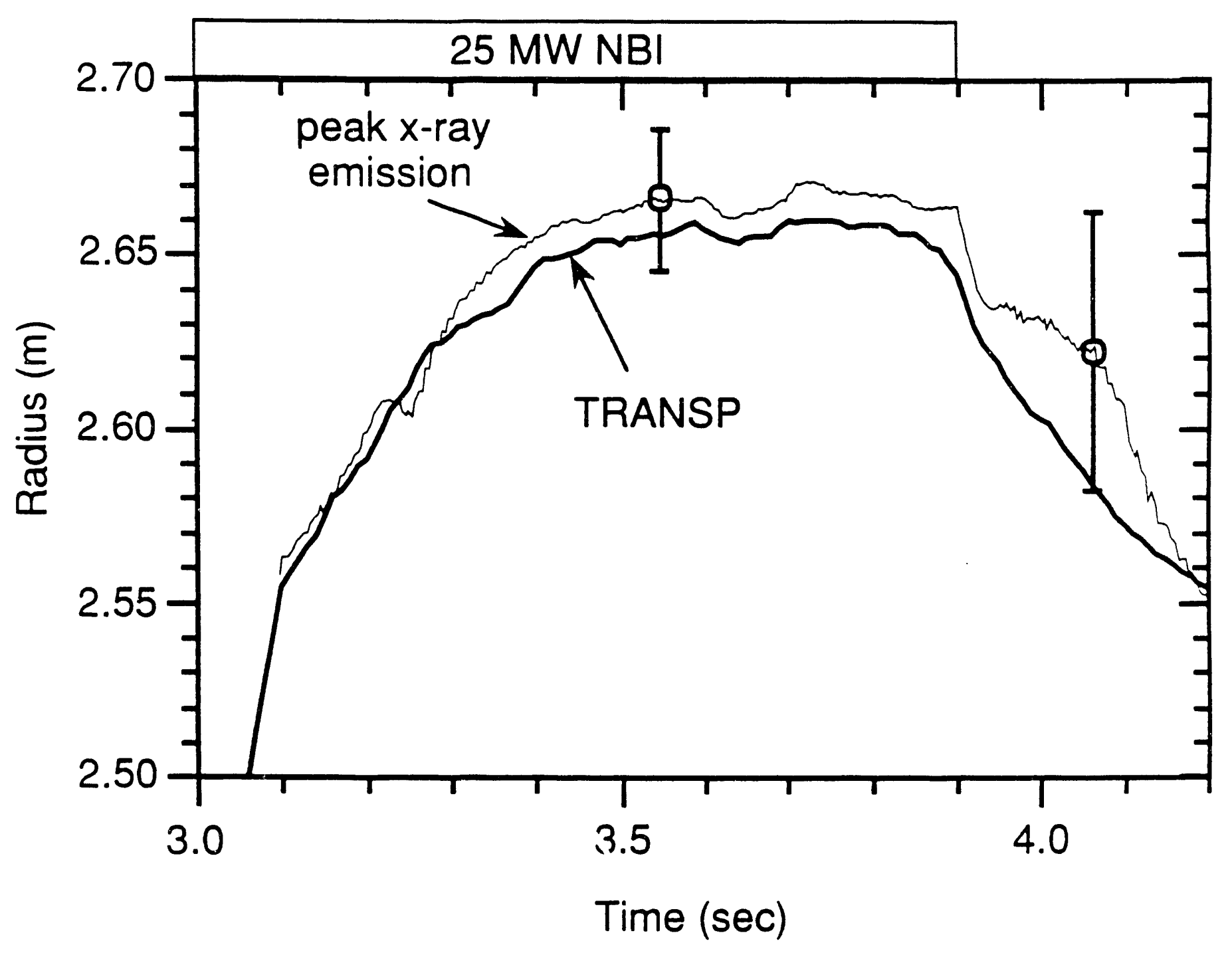

Fig $1 \mathrm{~J}$ 


\section{$q_{\psi}$ and shear profiles}

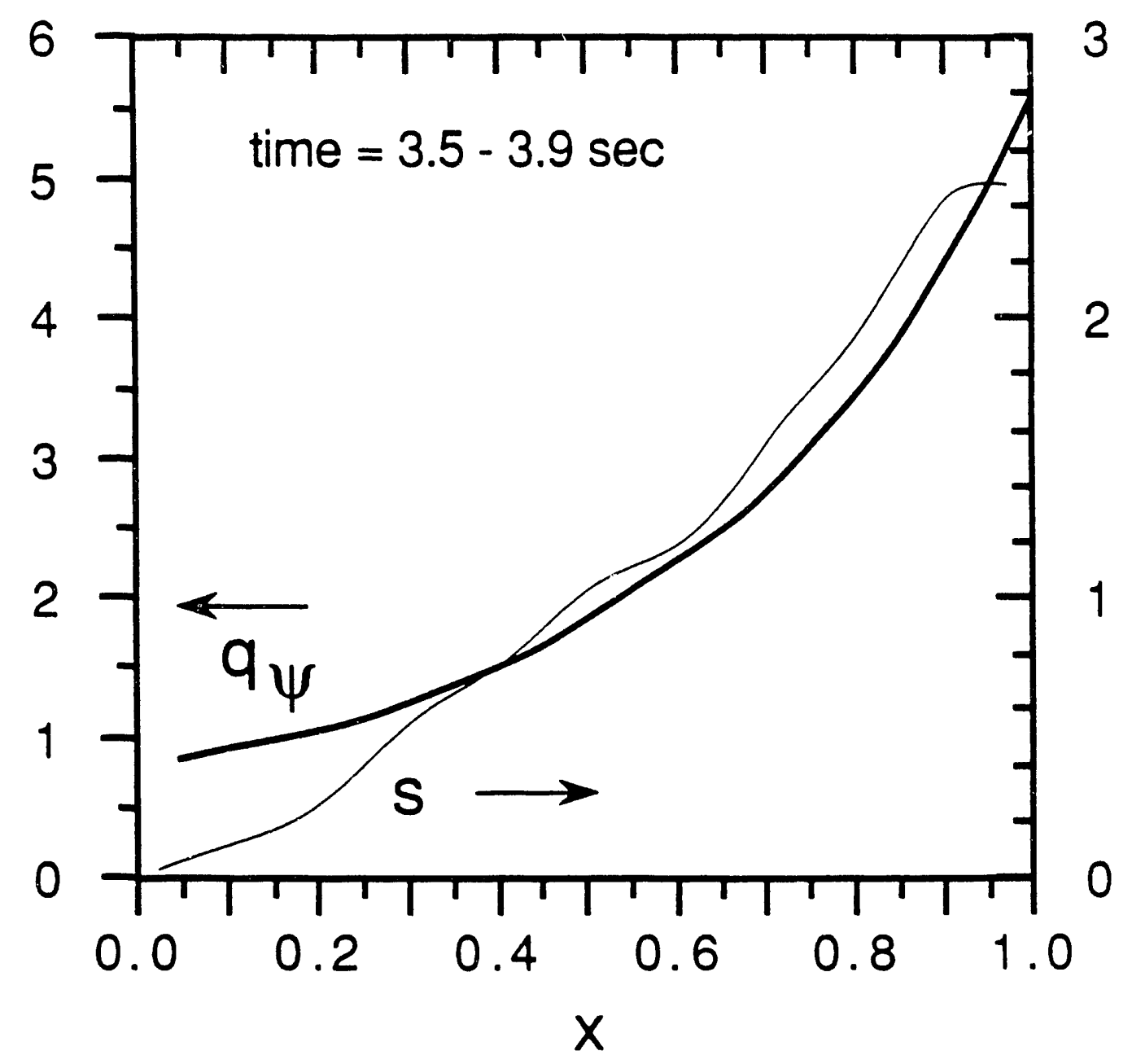

Fig $1 \mathrm{~K}$ 


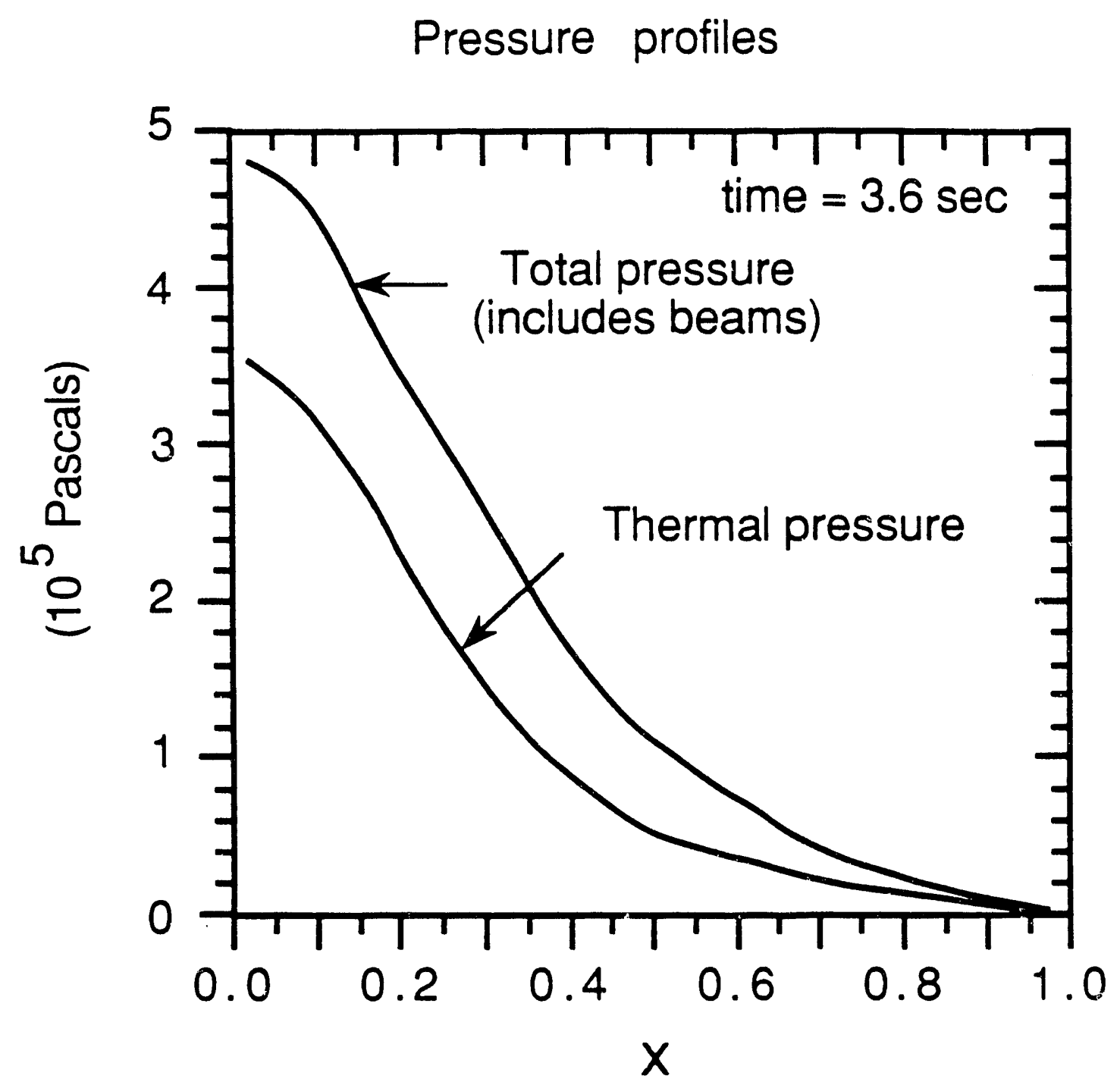

Fig 1L 


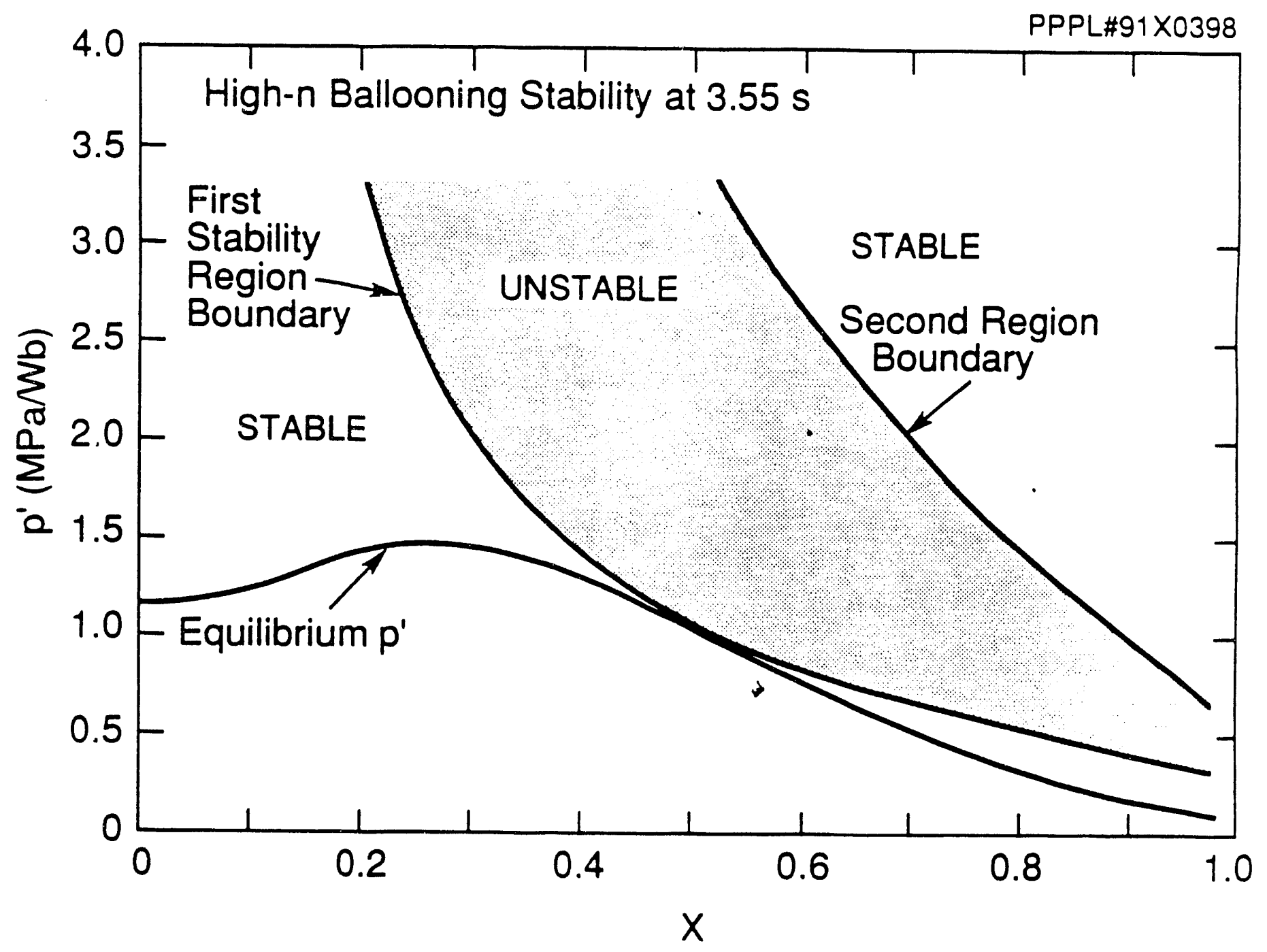

Fig 2 
Experimentally projected $Q_{D T}$

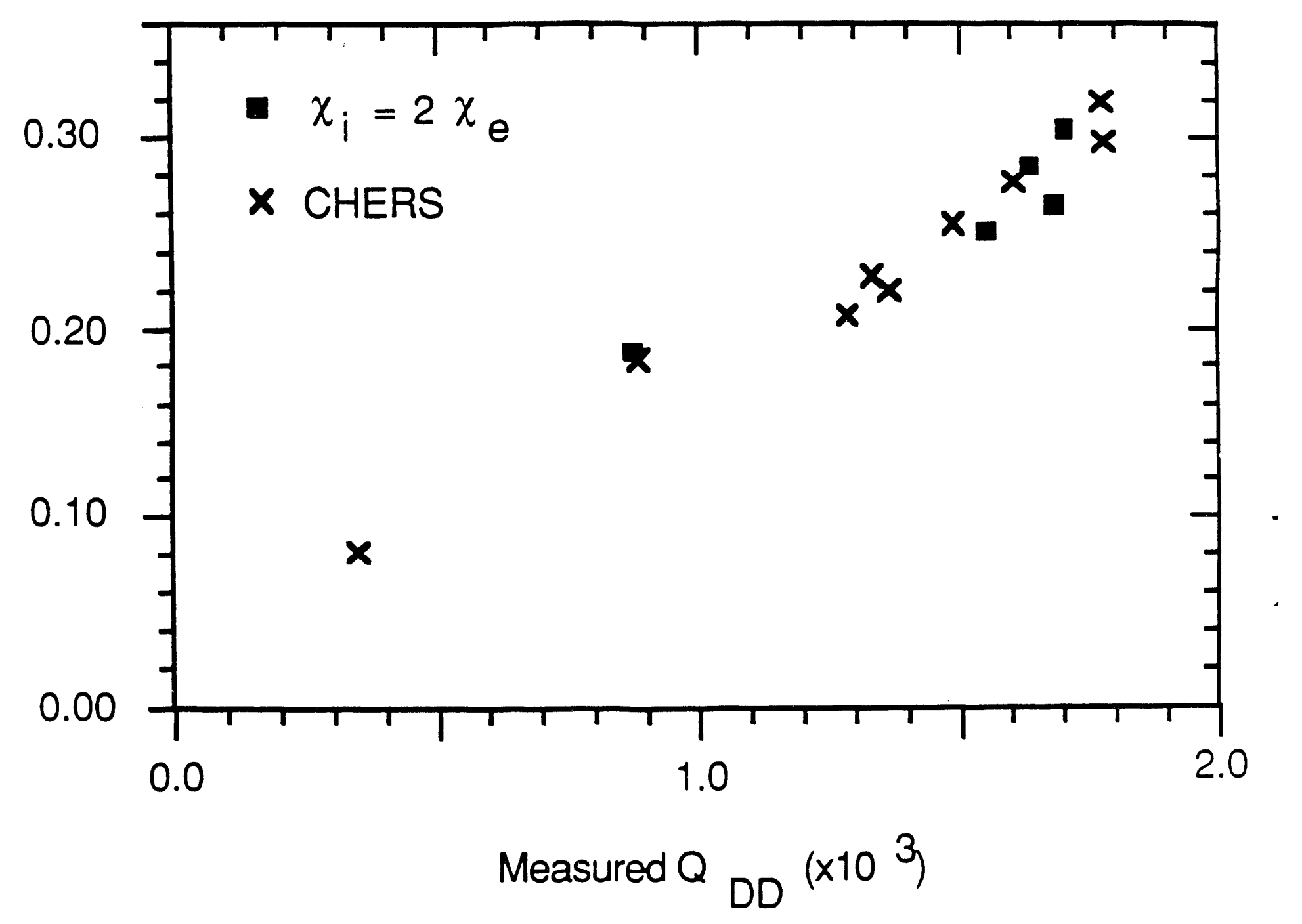

Fig 3 


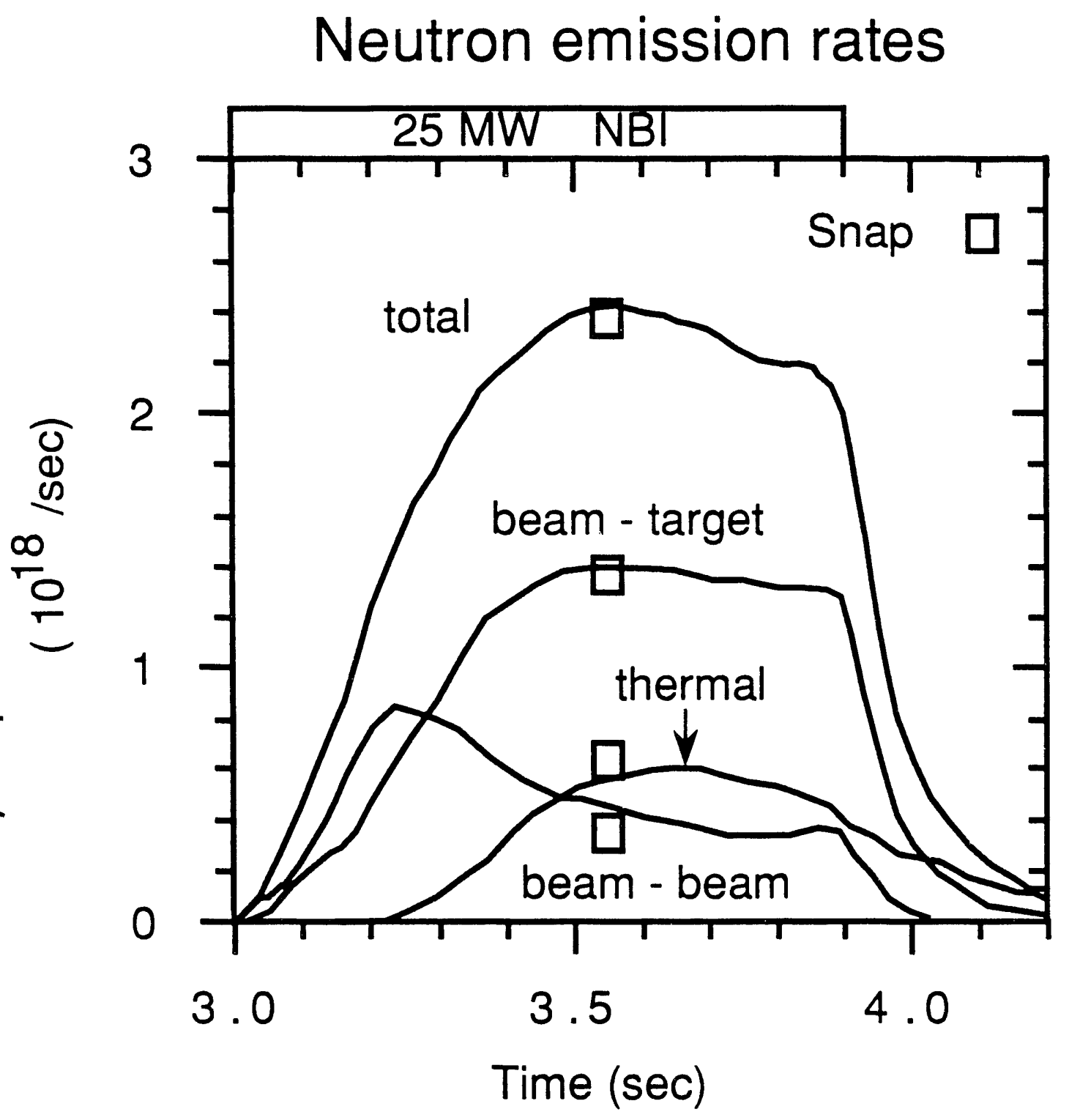

Fig 4A 
D-T neutron emission profiles

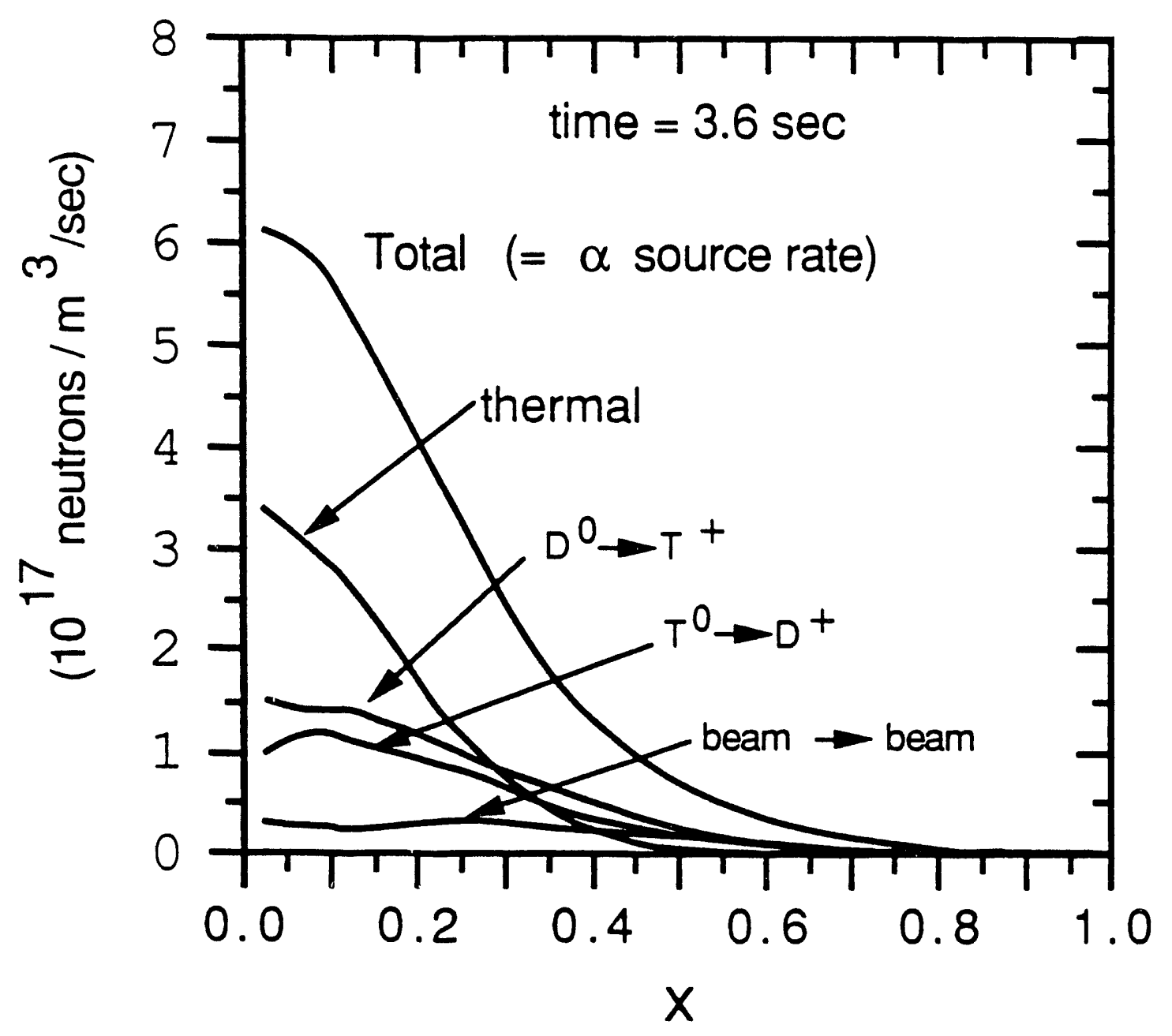

Fig 4B 


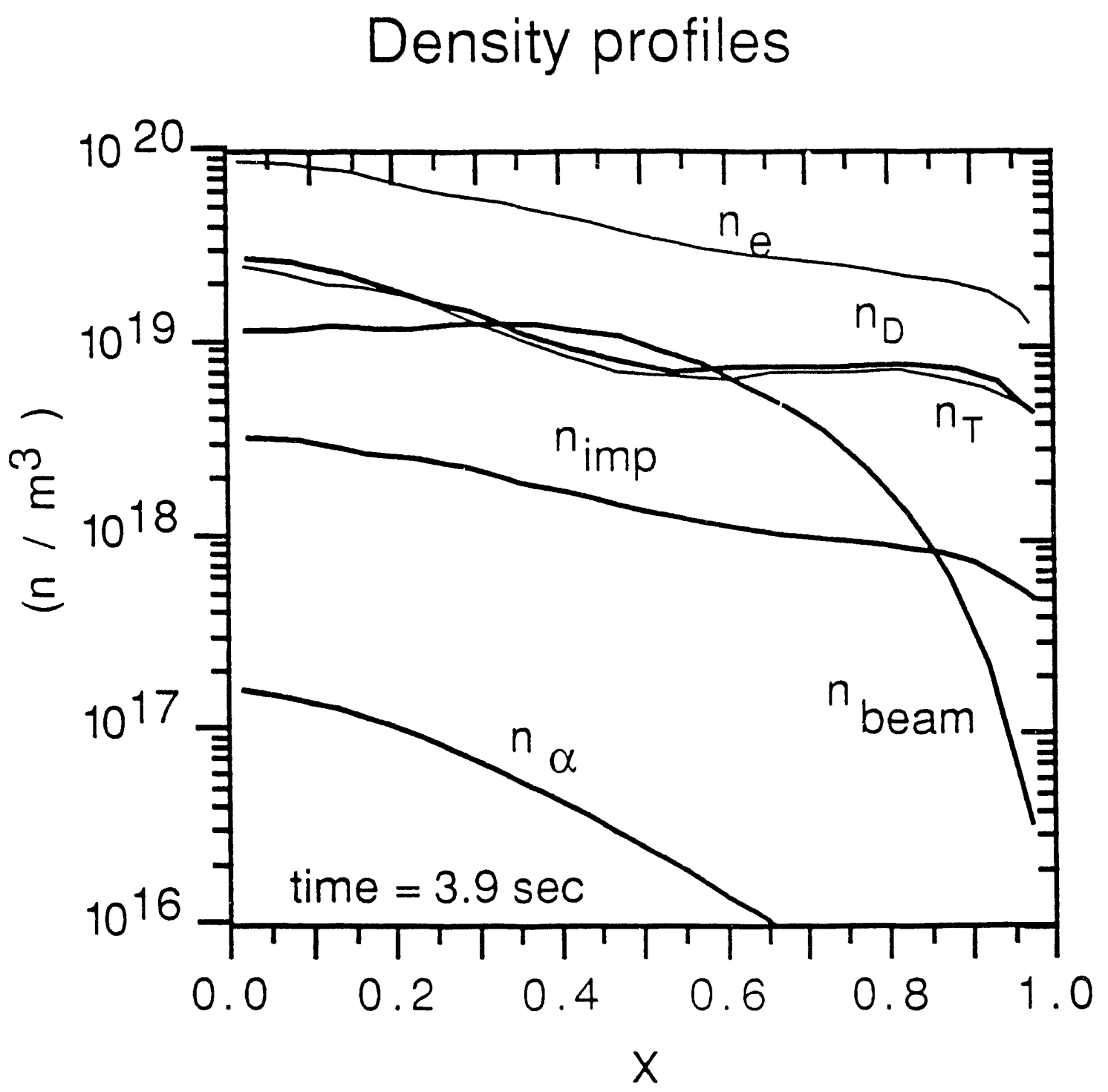

Fig 4C 


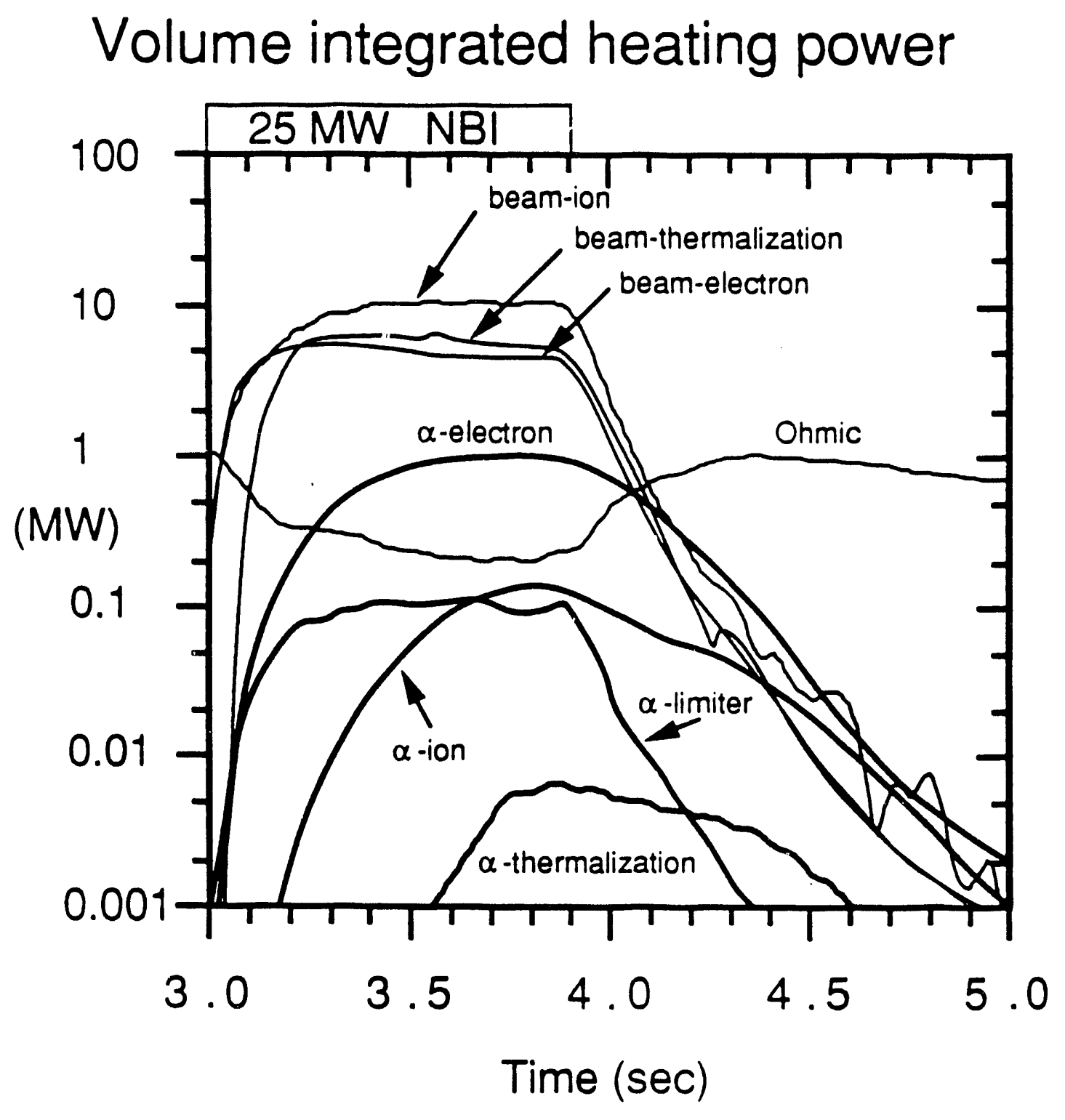

Fig 4D 
Ratios of $\alpha$ heating powers and losses

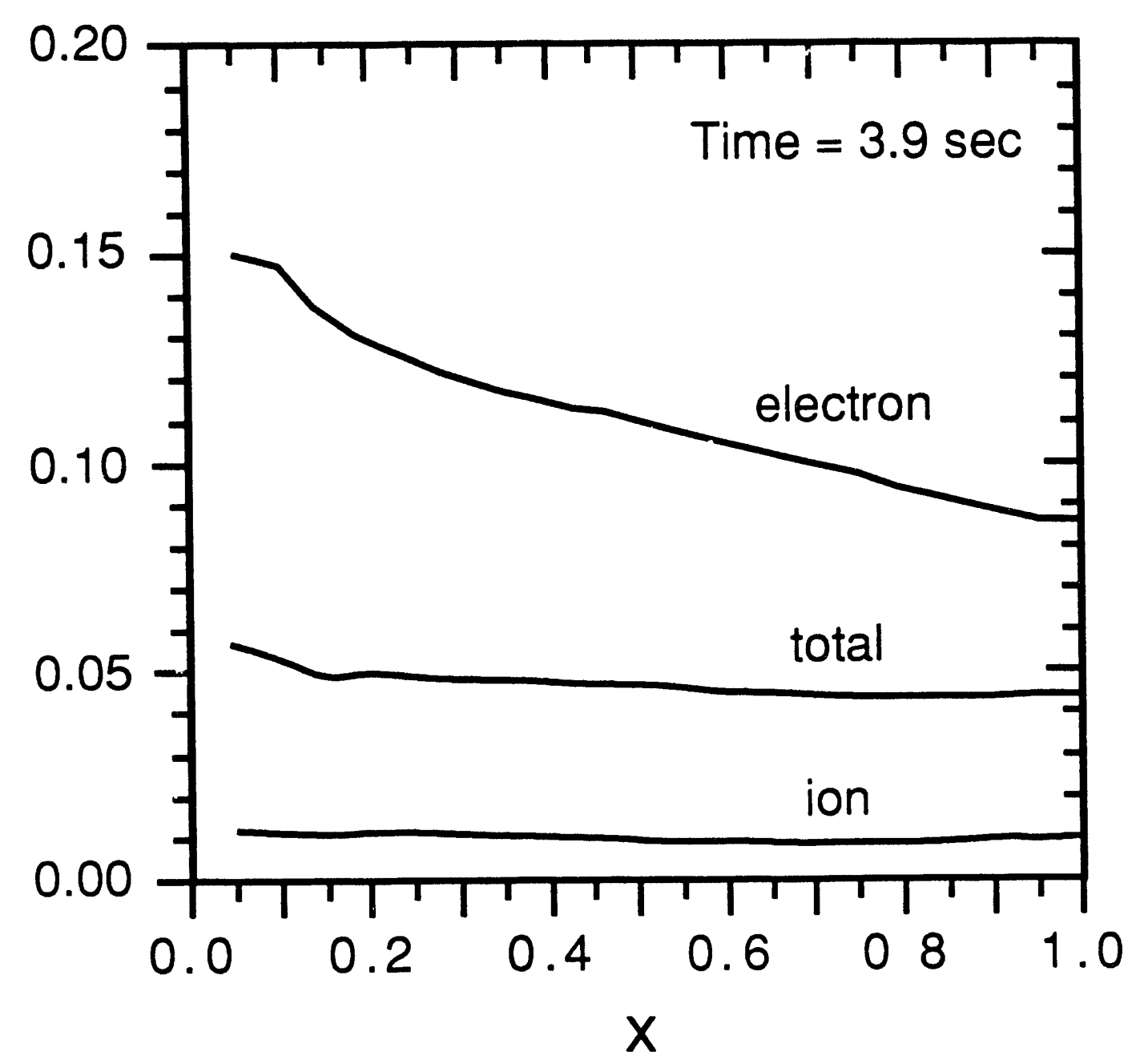

Fig 4E 
Central toroidal $\beta$

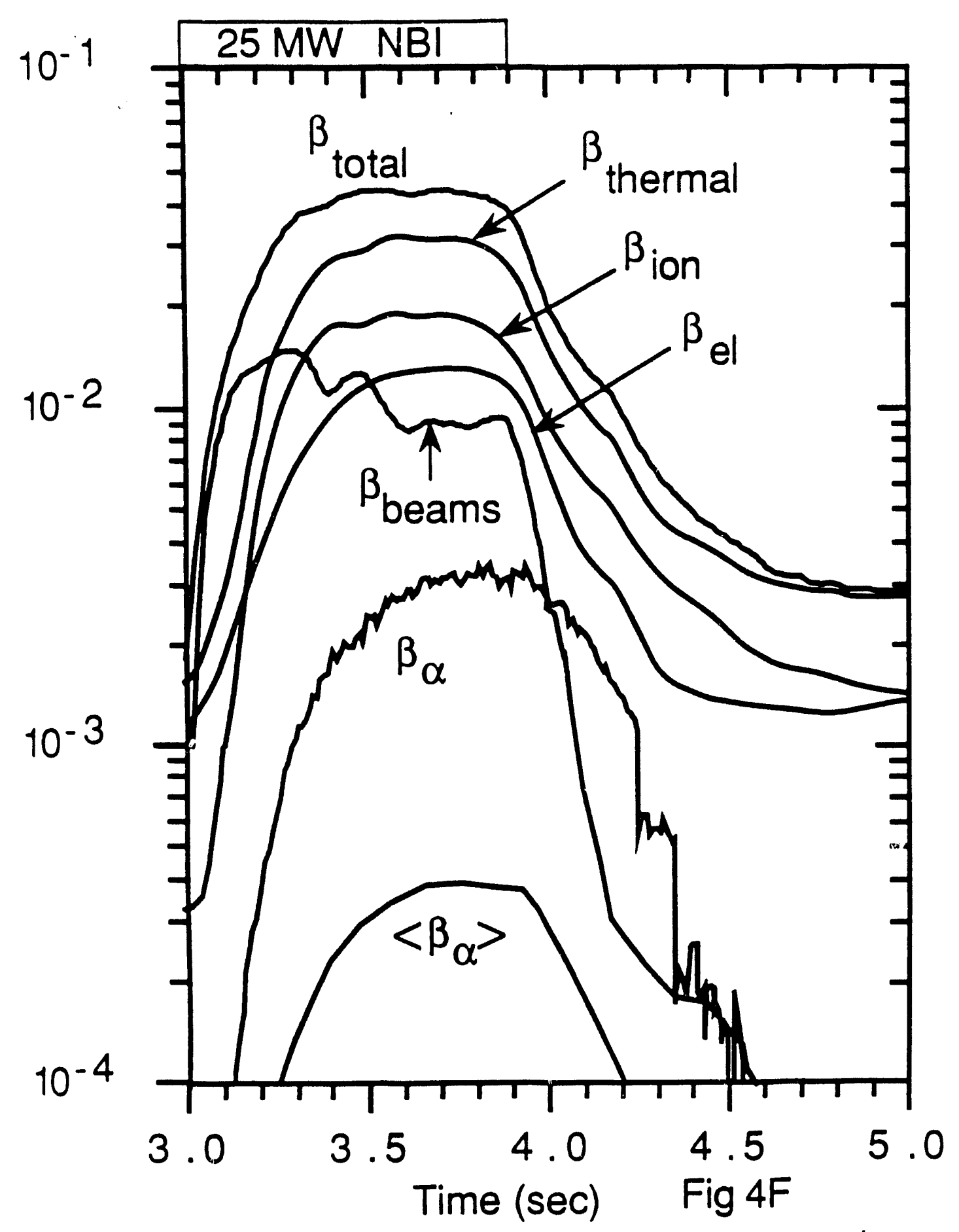




\section{Profiles of the contributions to $\beta$}

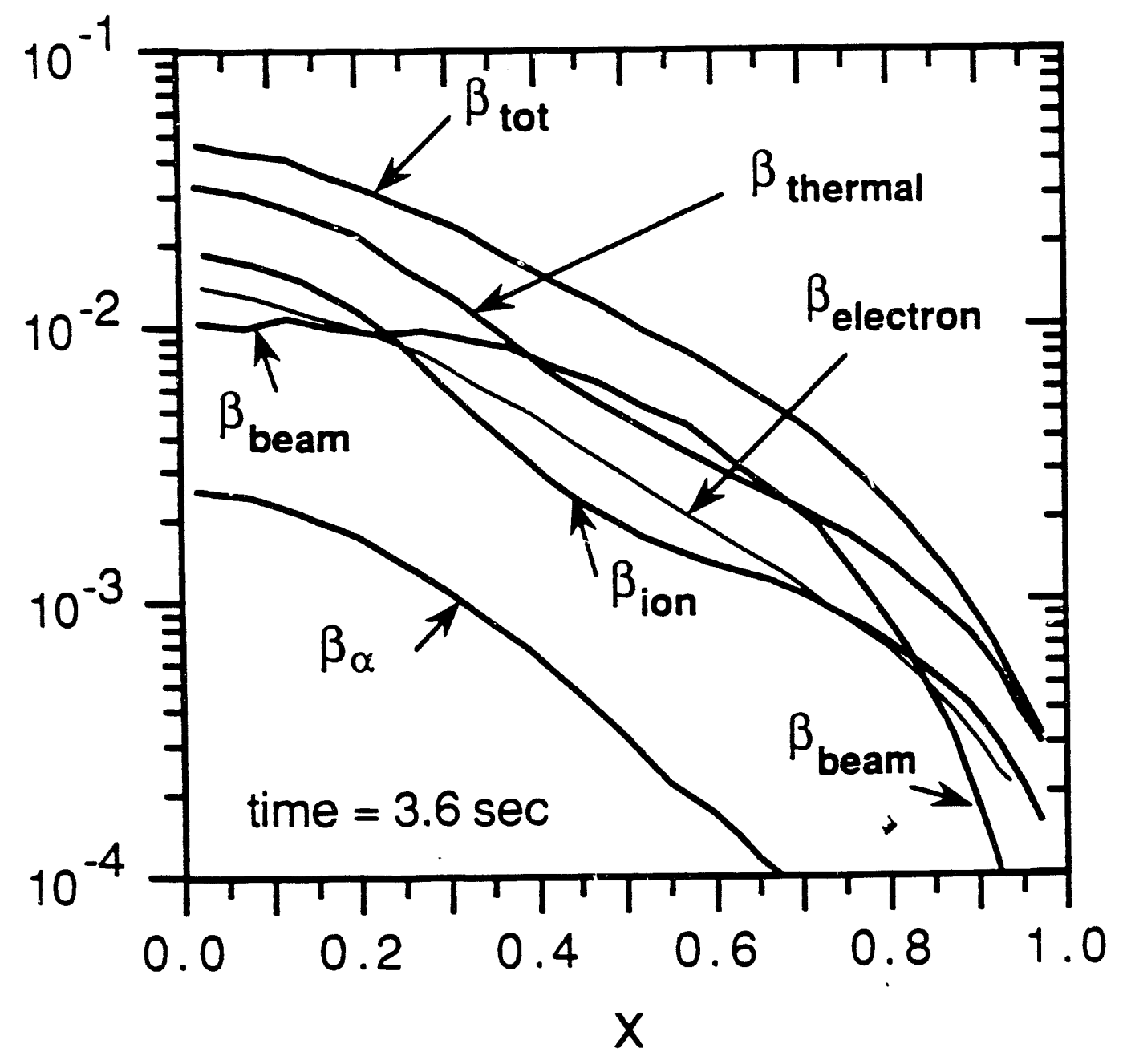

Fig 4G 
Relative $\alpha$ profiles

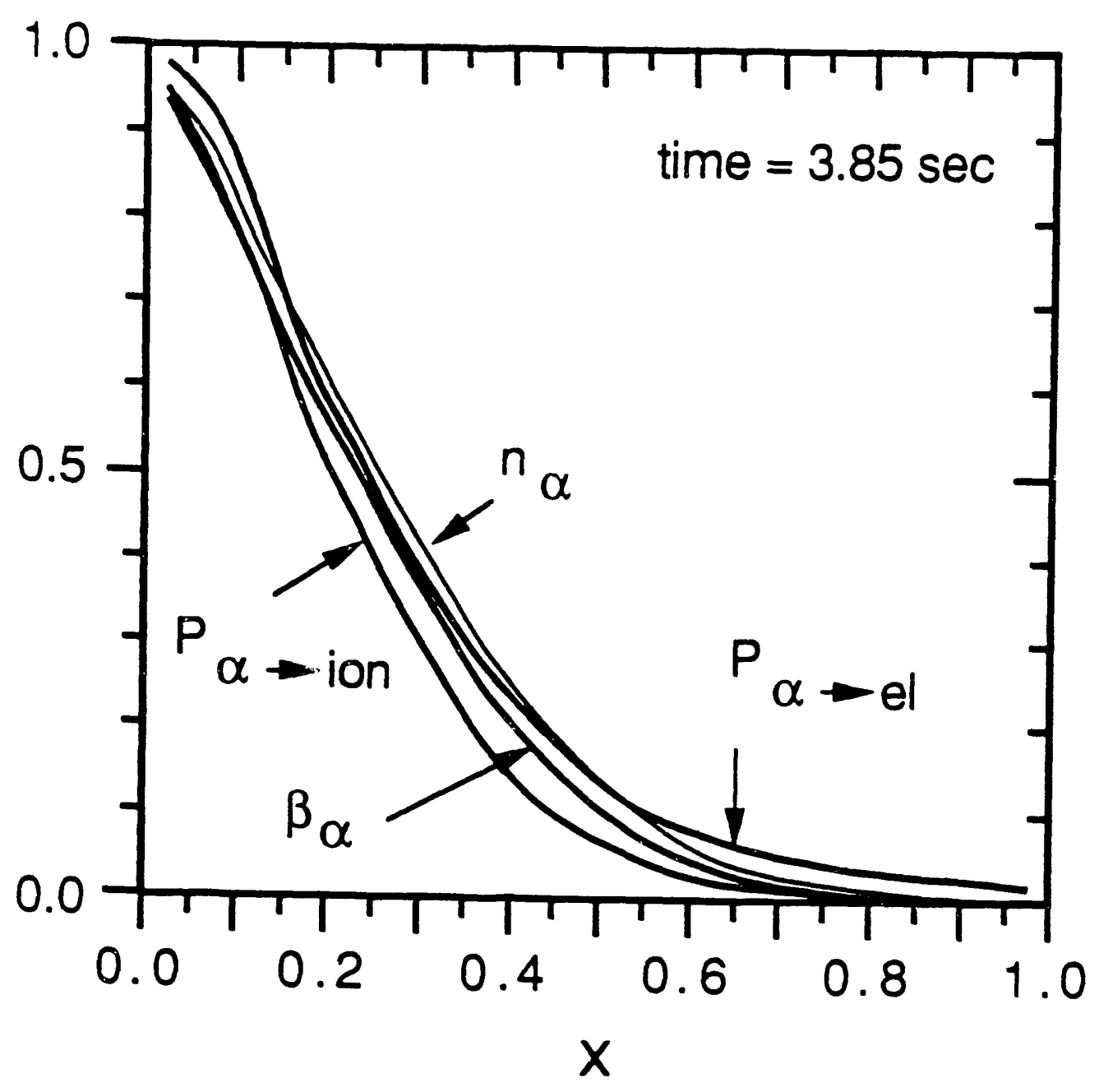

Fig 4H 
Average $\alpha$ energy and slowing down time

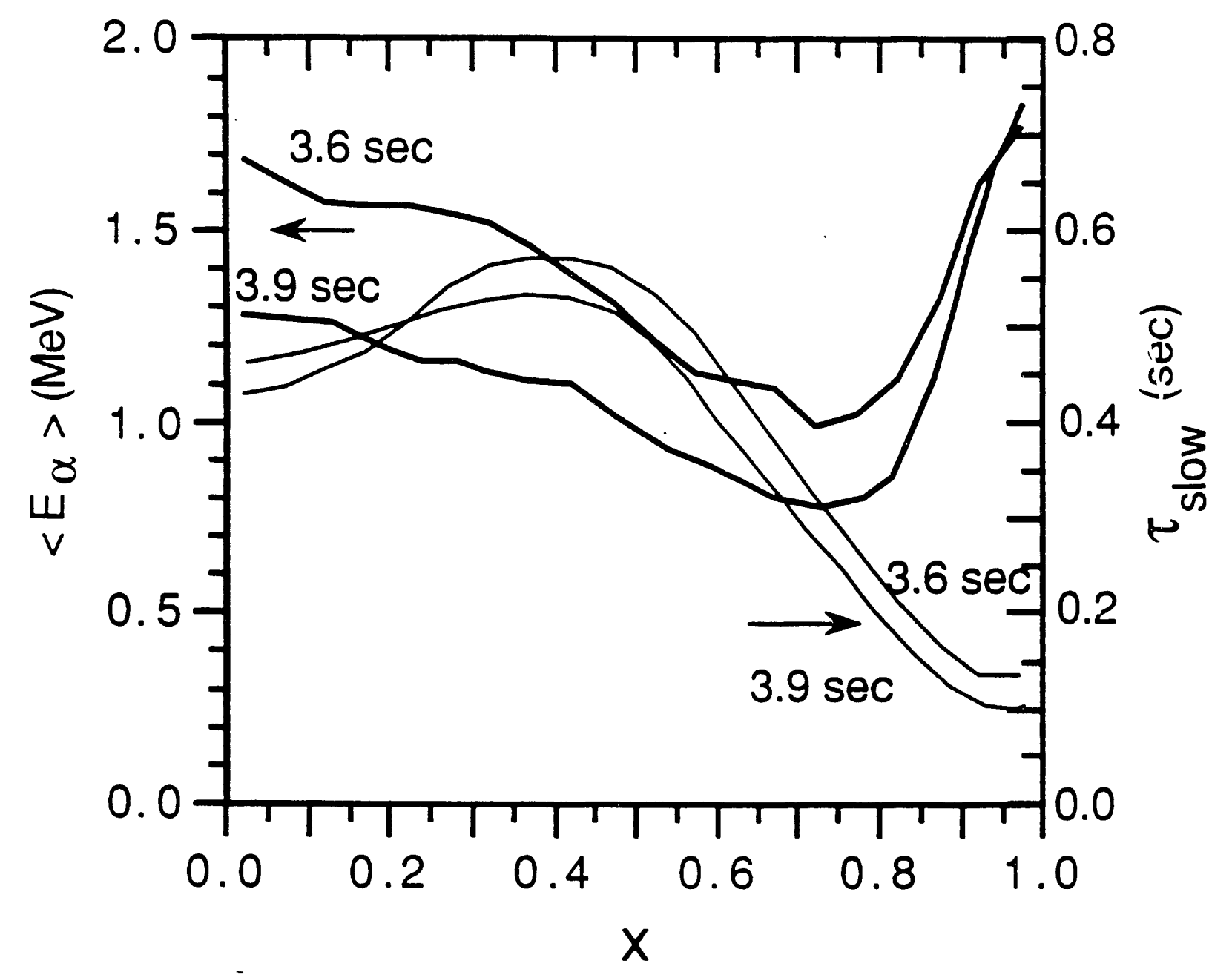

Fig 4 I 
Profiles of the Alfven frequency and speed

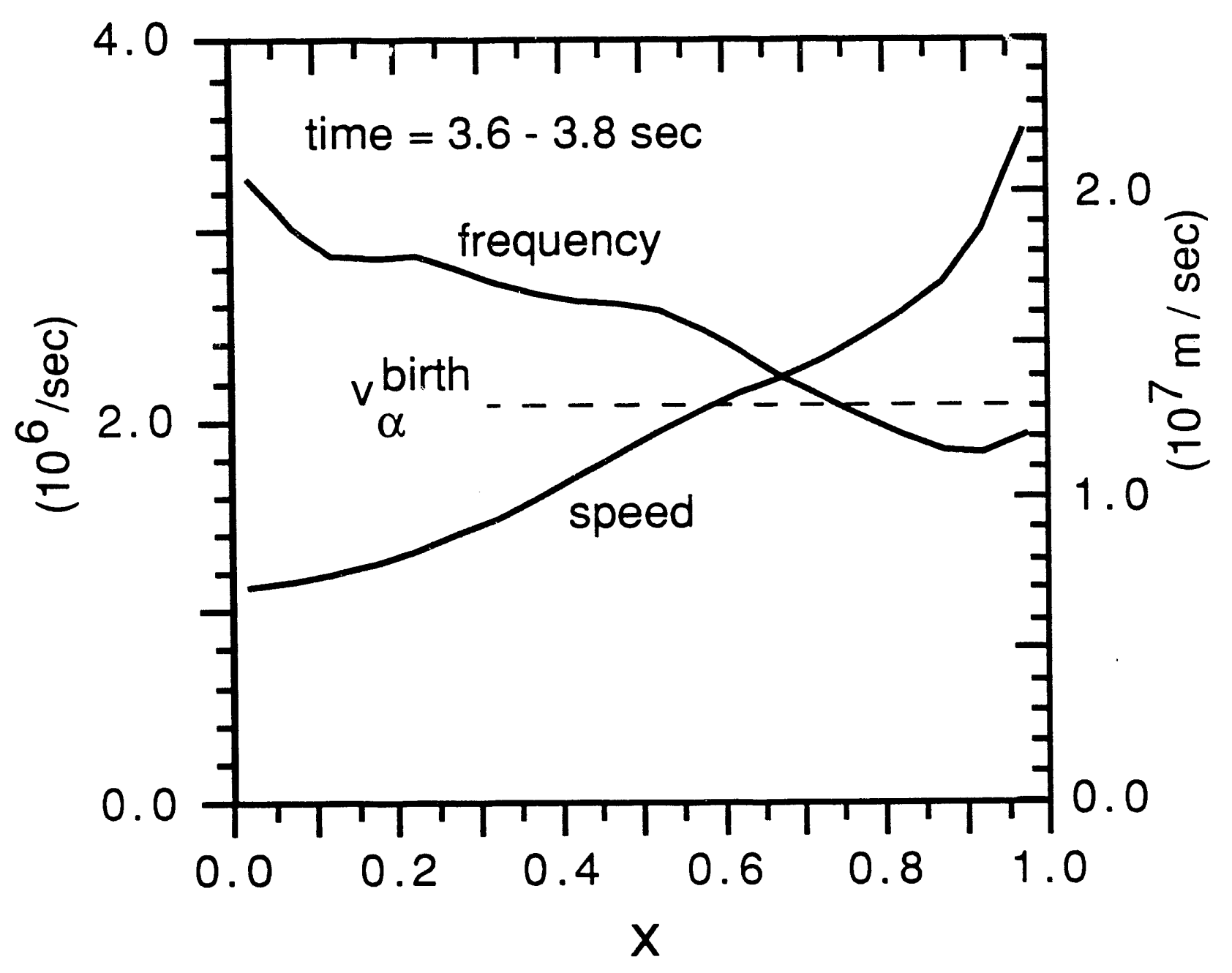

Fig 4J 
Ratio of the initial $\alpha$ and Alfvén speeds

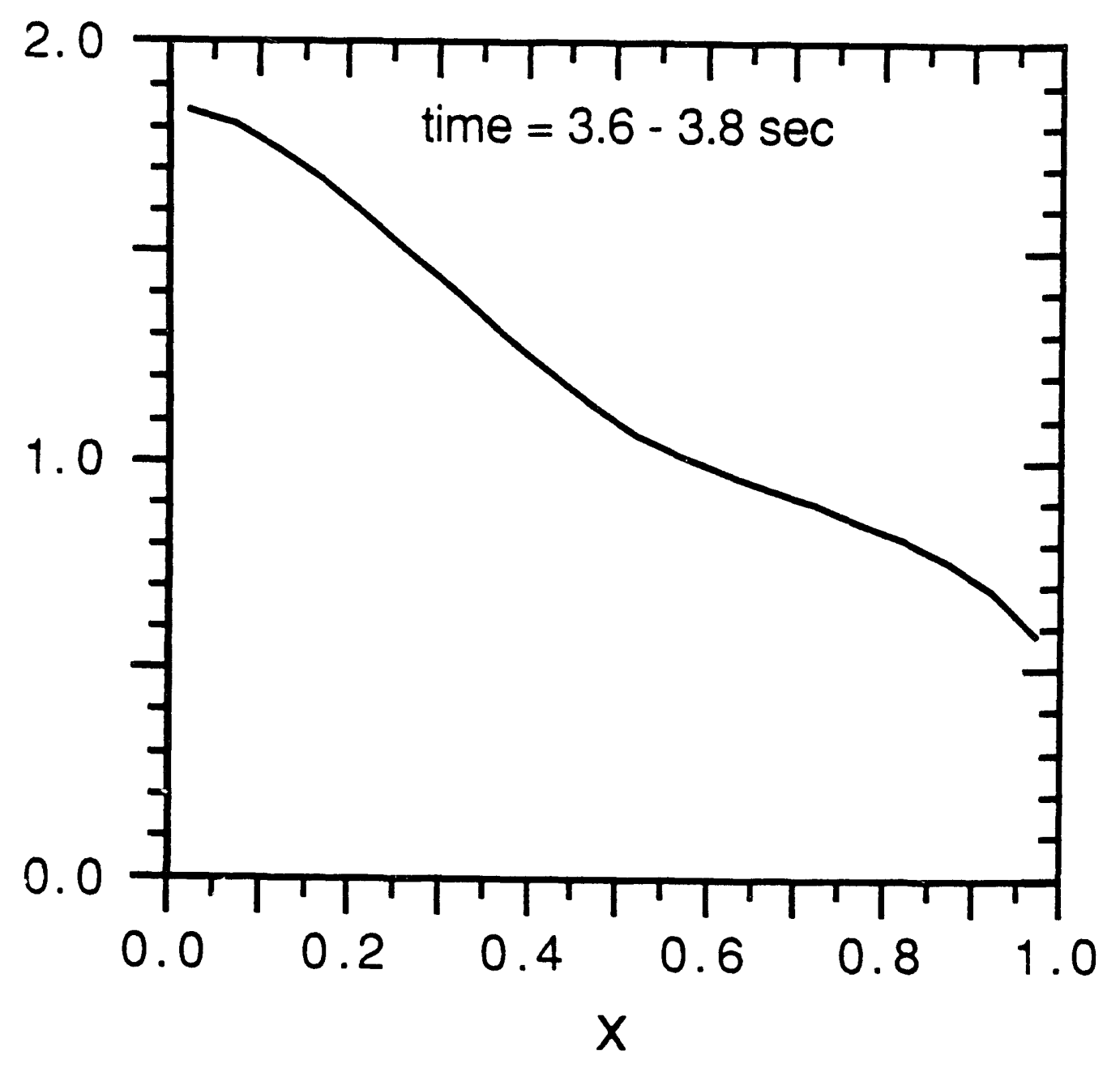

Fig 4K 
Ratio of $\alpha$ heating to beam and ion heating of electrons

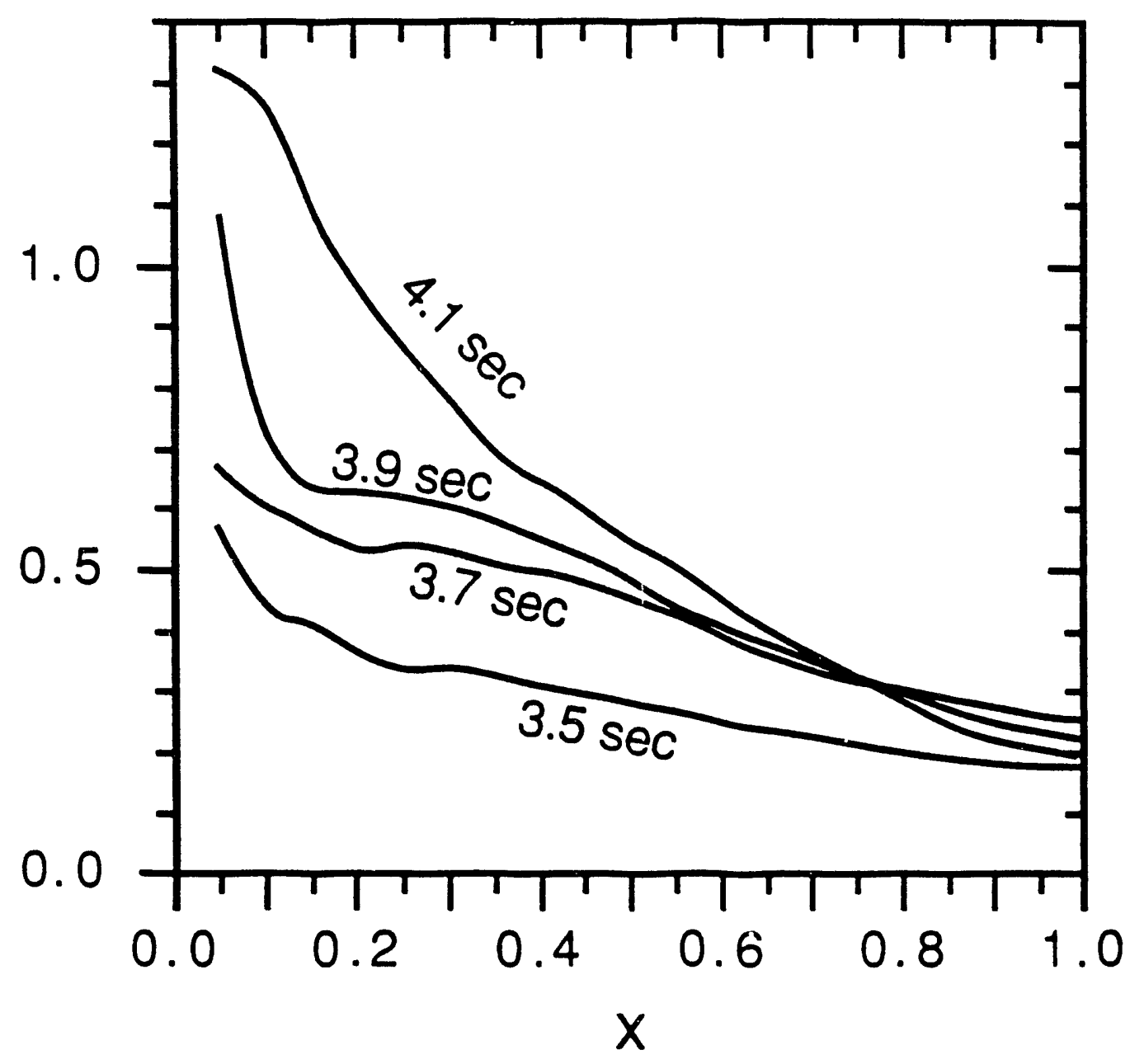

Fig $5 A$ 


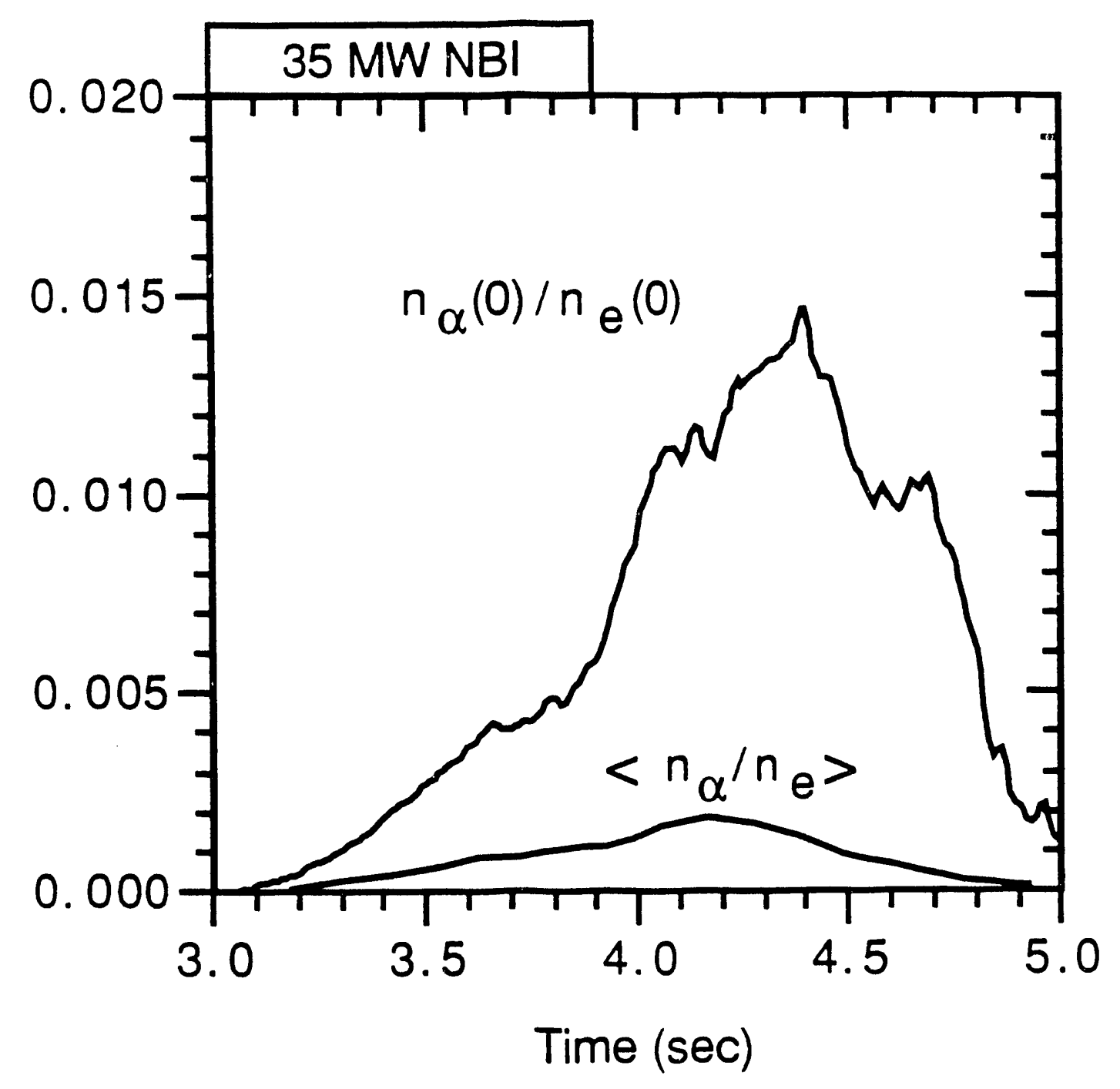

Fig 5B 


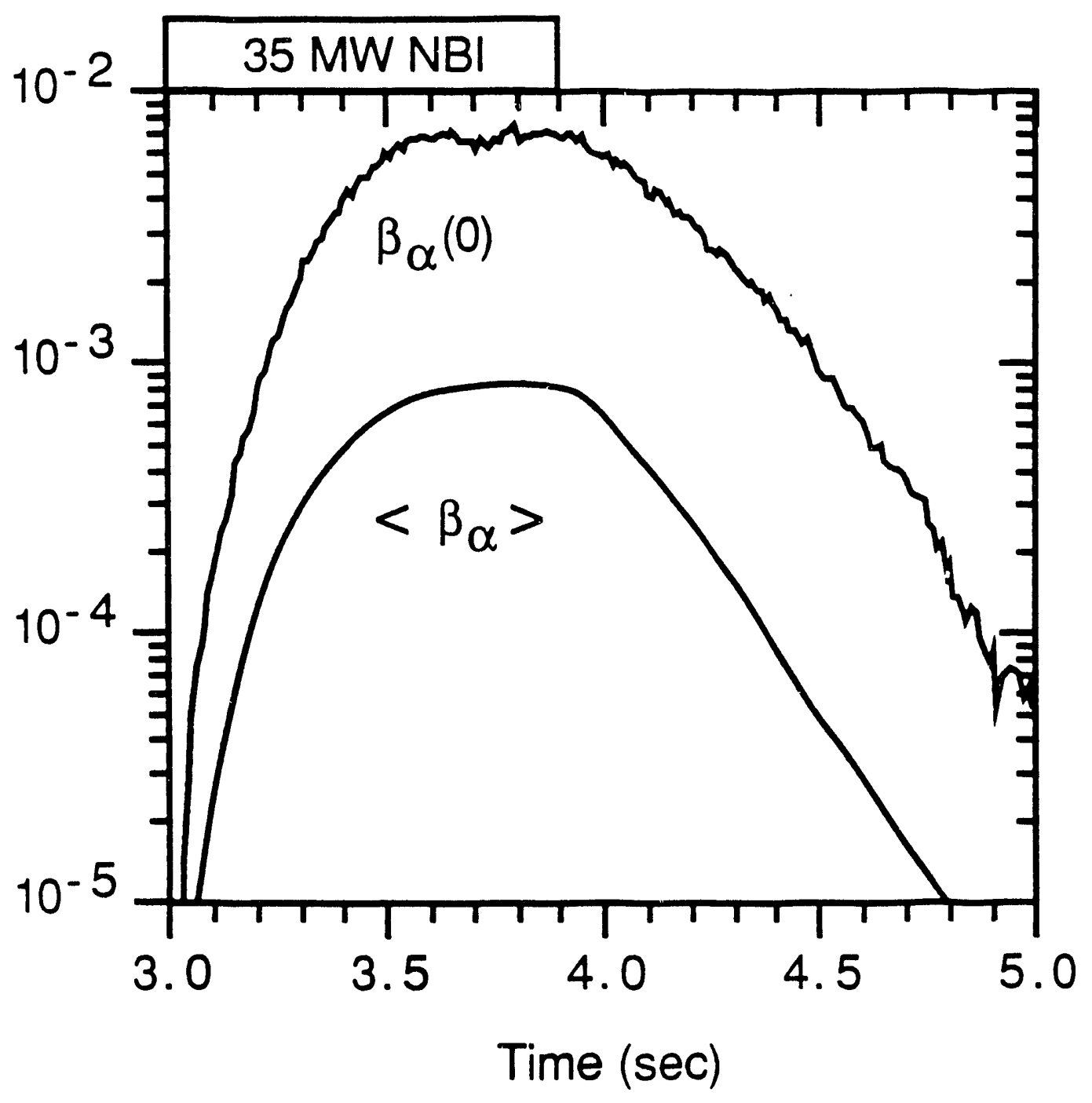

Fig $5 C$ 


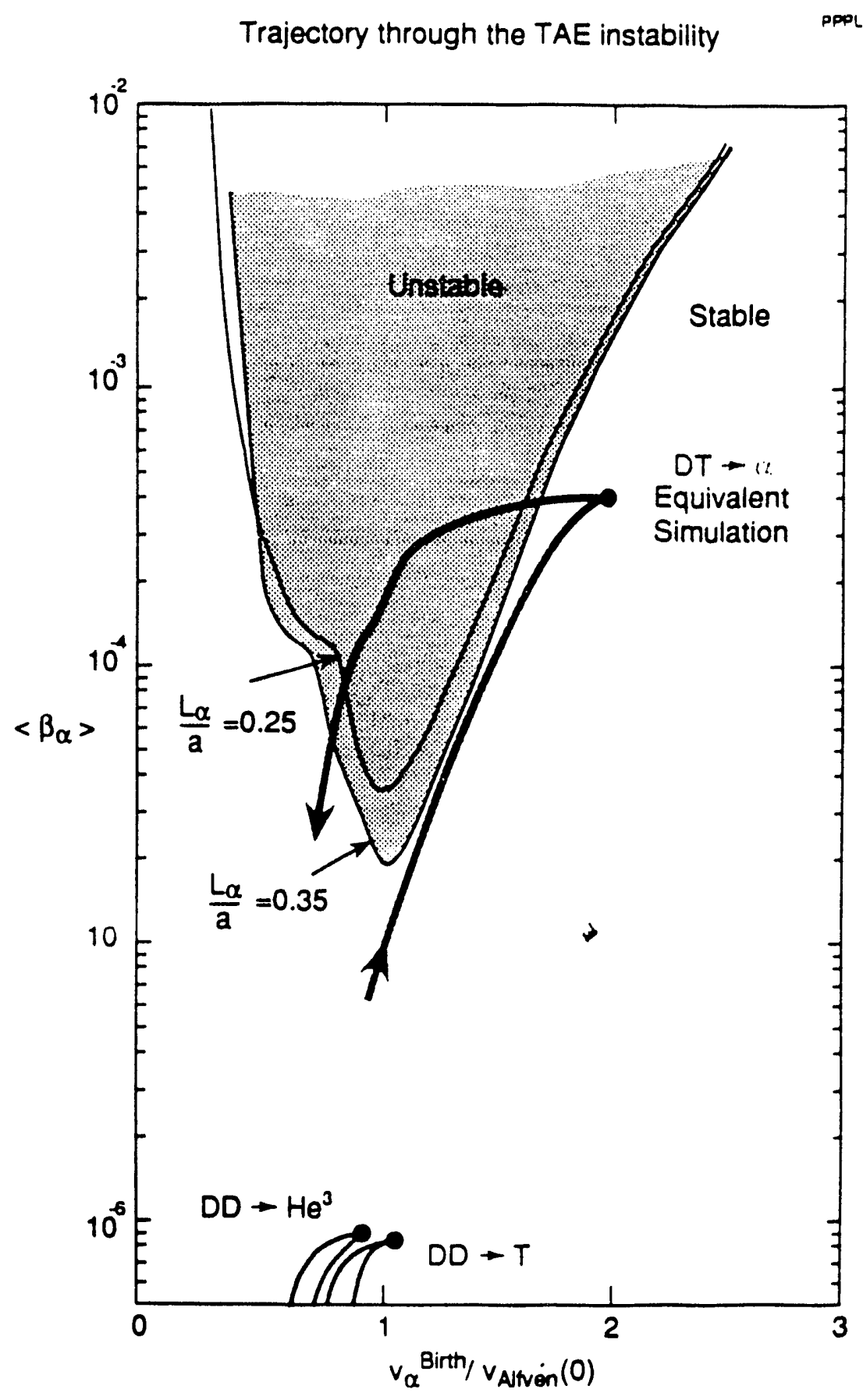

Fig 6 


\section{EXTERNAL DISTRIBUTION IN ADDITION TO UC-420}

Dr. F. Paoloni, Univ. of Wollongong, AUSTRALIA

Prot. M.H. Brennan, Univ. of Sydney, AUSTRALIA

Plasma Research Lab., Australian Nat. Univ., AUSTRALIA

Prof. I.R. Jones, Flinders Univ, AUSTRALIA

Prof. F. Cap, Inst. for Theoretical Physics, AUSTRIA

Prof. M. Heindler, Institut fur Theoretische Physik, AUSTRIA

Prof. M. Goossens, Astronomisch Instituut, BELGIUM

Ecole Royale Militairo, Lab. de Phy. Plasmas, BELGIUM

Commission-Europeen, DG. XII-Fusion Prog., BELGIUM

Prof. R. Boucique, Rijksuniversiteit Gent, BELGIUM

Dr. P.H. Sakanaka, Instituto Fisica, BPAZIL

Instituto Naciona Do Pesquiras Especiais-INPE, BRAZIL

Documents Office, Atomic Energy of Canada Lid., CANADA

Dr. M.P. Bachynski, MPB Technologies, Inc., CANADA

Dr. H.M. Skarsgard, Univ. of Sackatchewan, CANADA

Prof. J. Teichmann, Univ. of Montreal, CANADA

Prof. S.R. Sreenivasan, Univ. of Calgary, CANADA

Prof. T.W. Johnston, INRS-Energie, CANADA

Dr. R. Bolton, Contre canadien de fusion magnétique, CANADA

Dr. C.R. James, Univ. of Aberta, CANADA

Dr. P. Lukác, Komenskétho Universzita, CZECHO-SLOVAKIA

The Librarian, Cutham Laboratory. ENGLAND

Library, R61, Ruthertord Appleton Laboratory, ENGLAND

Mrs. S.A. Hutchinson, JET Library, ENGLAND

Dr. S.C. Shama, Univ. of South Pacific, FIJI ISLANDS

P. Mathonen, Univ. of Helsinki, FINLAND

Prof. M.N. Bussac, Ecole Polytechnique,, FRANCE

C. Mouttet, Lab. de Physique des Milieux lonisés, FRANCE

J. Radat, CENCADARACHE - Bat 506, FRANCE

Prof. E. Economou, Univ. of Crete, GREECE

Ms. C. Rinni, Univ. of loannina, GREECE

Dr. T. Mued, Academy Bibliographic Ser., HONG KONG

Preprint Library, Hungarian Academy of Sci., HUNGAPY

Dr. B. DasGupta, Saha inst. of Nuctear Physics, INDIA

Dr. P. Kaw, Inst. tor Plasma Research, INDIA

Dr. P. Rosenau, Israel inst. of Technology, ISPAEL

Libravian, International Conter for Theo Physics, ITALY

Miss C. De Palo, Associazione EURATOM-ENEA, ITALY

Dr. G. Grosso, Istituto di Fisica del Plasma, ITALY

Prof. G. Rostangni, Istituto Gas lonizzati Dol Cnr, ITALY

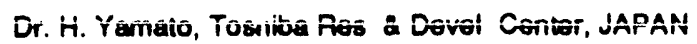

Prof. I. Kawakami, Hiroshima Univ., JAPAN

Prof. K. Nishikawa, Hiroshima Univ., JAPAN

Director, Japan Atomic Energy Research Inst., JAPAN

Prof. S. Itoh, Kyusthu Univ., JAPAN

Research Into. Cr., National Instit. for Fusion Science. JAPAN

Prof. S. Tanaka, Kyoto Univ., JAPAN

Library, Kyoto Univ., JAPAN

Prot. N. Inowe, Univ. of Tokyo, JAPAN

Secrotary, Plasma Section, Electrotochnical Lab., JAPAN

S. Mori, Tectnicel Advisor, JAERI, JAPAN

Dr. O. Miterai, Kumamoto inst. of Technology, JAPAN

J. Hyeon-Sook, Korea Alomic Energy Research inst. KOREA

D.I. Choi, The Korea Adv. Inst of Sai. \& Tech., KOREA

Prof. B.S. Liloy, Univ. of Waikato, NEW ZEALAND

Inst of Physics, Chinese Acad Sci PEOPLE'S REP. OF CHINA

Library, Inst. of Plasma Physics, PEOPLE'S REP. OF CHINA

Tsinghua Univ. Library, PEOPLE'S REPUBLIC OF CHINA

Z. L. S.W. Inst Physica, PEOPLE'S REPUBLIC OF CHINA

Prof. J.A.C. Cabral, Instituto Superior Tecnico, PORTUGAL

Dr. O. Petrus, AL I CUZA Univ., ROMANIA

Dr. J. de Villiers, Fusion Studies, AEC, S. AFRICA

Prof. M.A. Hellberg. Univ. of Natal, S. AFRICA

Prof. D.E. Kim, Pohang Inst. of Sci. \& Tech., SO. KOREA

Prof. C.I.E.M.A.T, Fusion Division Library, SPAIN

Dr. L Stonfio, Univ. of UMEA, SWEDEN

Library, Royad Inst. of Technology, SWEDEN

Prot. H. Wilholmson, Chaimers Univ. of Tech., SWEDEN

Contre Phys. Des Plasmas, Ecole Polytech, SWITZERLAND

Bibliotheok, Inst. Voor Plasma-Fysice. THE NETHERLANDS

Asst. Prof. Dr. S. Cakir, Middle East Tect. Univ., TURKEY

Dr. V.A. Gukhikh,Sci. Ros. Inst. Electrophys.I Apparatus, USSR

Dr. D.D. Ayutov, Siberian Branch of Academy of Sa., USSR

Dr. G.A. Eliseov, I.V. Kurchatov Inst. USSR

Librarian. The Ukr.SSA Academy of Sciences, USSR

Dr. LM. Kovrizhnykh, Inst. of General Physics, USSR

Kemforschungsanlage GmbH, Zentralbibliothek, W. GERMANY

Bibliothek, Inst. For Plasmatorscting, W. GERMANY

Prof. K. Schinder, Ruhr-Universitát Bochum, W. GERMANY

Dr. F. Wagner, (ASDEX), Max-Planck-Institut, W. GERMANY

Librarian, Max-Planck-Institur, W. GERMANY

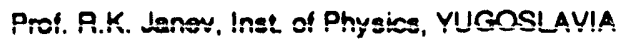



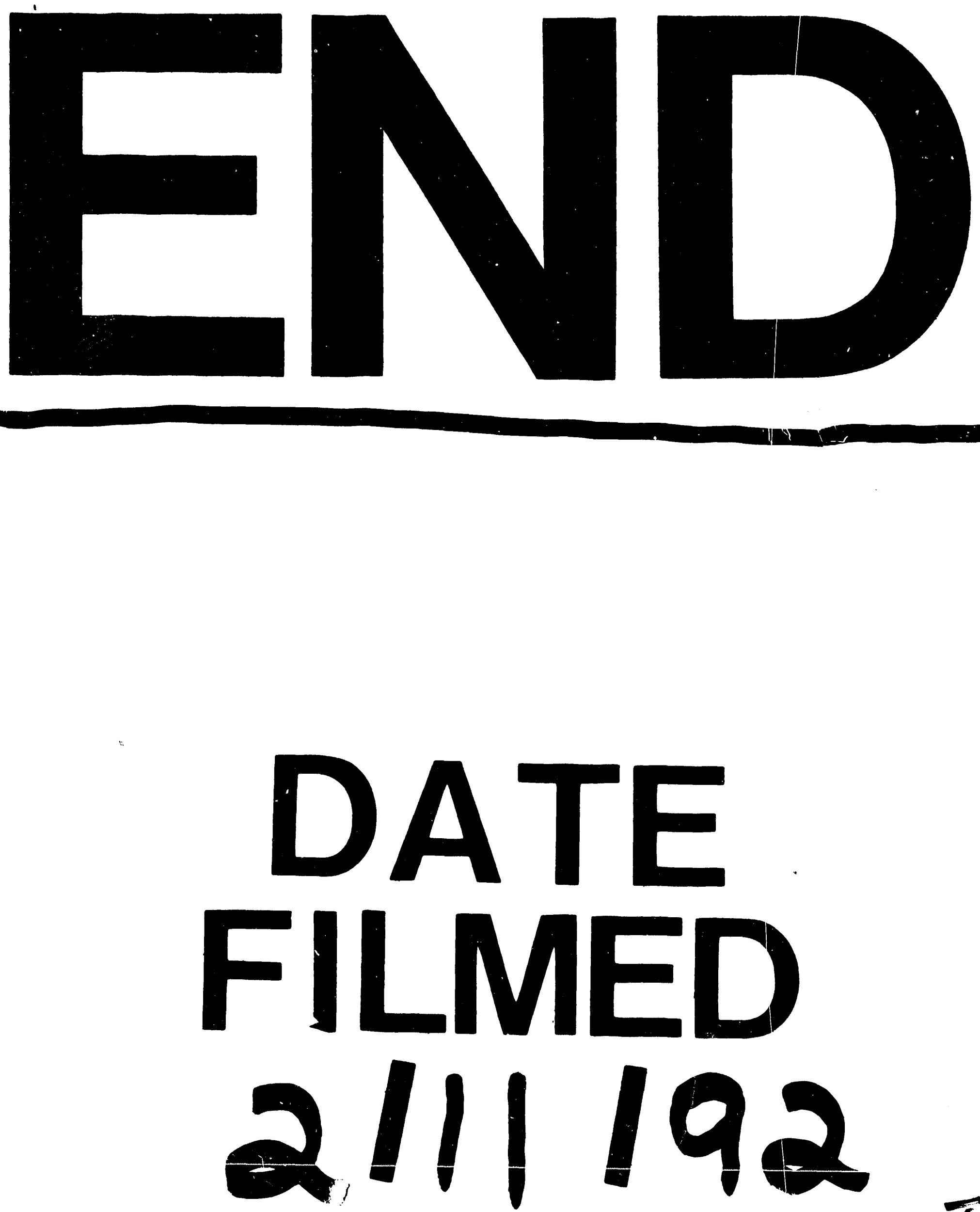

$I$ 


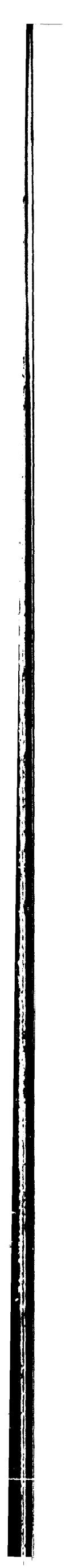

